\title{
Cambio tecnológico, PRODUCTIVIdAd Y PRODUCTO DE LARGO PLAZO DE PERÚ
}

\author{
Félix Jiménez ${ }^{\mathrm{a}}$
}

Fecha de recepción: 15 de noviembre de 2019. Fecha de aceptación: 15 de junio de 2020.

$$
\text { https://doi.org/10.22201/iiec.20078951e.2021.Especial.69802 }
$$

Resumen. Este artículo presenta las estimaciones del producto de largo plazo corres-
pondientes a dos enfoques teóricos. El primero es el enfoque del crecimiento dirigido
por la demanda. Se estiman dos modelos de producción de largo plazo: el de Shaikh y
Moudud (2004) con una modificación para captar el cambio técnico determinado por
la demanda en el sentido kaldoriano (Kaldor, 1957); y el de Thirlwall (1979), según el
cual las tasas teóricas de crecimiento de largo plazo de la economía están determinadas
por las tasas de crecimiento de las exportaciones. El segundo enfoque es el neoclásico
de Solow (1956) y Swan (1956). Para este enfoque el crecimiento de largo plazo está
limitado por factores de oferta (los factores de producción capital y trabajo). Palabras clave: producto de largo plazo; productividad; cambio técnico; demanda; capital y trabajo producto potencial.

Clasificación JEL: B23; D24; E12; E13; E23; O47.

\section{Technological Change, Productivity AND PERU'S LONG-TERM OUTPUT}

\begin{abstract}
This article estimates long-term output using two distinct theoretical approaches. The first is the demand-led growth approach. In this approach, two long-term output models are estimated: that of Shaikh and Moudud (2004), with a modification to capture demand-driven technical change in the Kaldorian sense (Kaldor, 1957); and that of Thirlwall (1979), according to which the theoretical long-term growth rates of the economy are determined by export growth rates. The second approach is the neoclassical approach of Solow (1956) and Swan (1956). In this approach, long-term growth is limited by supply factors (the factors of production capital and labor).
\end{abstract}

Key Words: long-term output; productivity; technical change; demand; capital and labor; potential output.

\footnotetext{
${ }^{a}$ Pontificia Universidad Católica del Perú, Perú. Correo electrónico: fjimene@pucp.edu.pe
} 


\section{INTRODUCCIÓN}

Las consecuencias de la crisis financiera global de 2008 han originado un intenso debate sobre los efectos de los shocks adversos en el Producto Interno Bruto (Рів) de largo plazo, más conocido como Producto Potencial. Diversas investigaciones para los países avanzados muestran que las recesiones y políticas macroeconómicas recesivas impactan negativamente en dicho producto y que las brechas se cierran con la revisión hacia abajo del producto potencial. No se cumple la idea de que el PIB retorna a su nivel potencial previo al shock. Los shocks tienen efectos permanentes. Por lo tanto, se puede decir que los shocks adversos de demanda tienen efectos permanentes, ya que impactan en el producto de largo plazo (Haltmaier, 2012; Reifschneider et al., 2013; Ball, 2014; Fatás y Summers, 2015; Martin et al., 2015).

Los economistas de la corriente convencional sostienen que, al quitarle al producto su tendencia de largo plazo lo que queda es un proceso puramente estacionario. Esta idea es parte de la teoría macroeconómica dominante desde inicios de la década de 1980, según la cual las políticas de administración de la demanda no afectan el nivel de producción de largo plazo (De Long y Summers, 1988). Como apunta Blanchard comentando críticamente la concepción sobre las fluctuaciones de esa teoría: "El futuro esperado depende en parte de las decisiones actuales o corrientes. [...] Pequeñas perturbaciones [exógenas o de política] pueden tener grandes efectos adversos o podrían dar lugar a depresiones largas y persistentes" (2014, pp. 28 y 29).

Una de las razones que explican por qué las recesiones -y, en general, las políticas económicas- impactan en el producto de largo plazo, es la caída de las inversiones. La consecuente desaceleración de la acumulación de capital impacta negativamente en el crecimiento de la capacidad productiva y, por lo tanto, del producto de largo plazo (Ball, 2014). Además, se sabe que las políticas económicas pueden retrasar o acelerar el crecimiento económico y, así, determinar el comportamiento del producto de largo plazo. La década perdida de América Latina y, en particular, de Perú -la década de 1980-, es un ejemplo de esta afirmación. Las políticas de austeridad que privilegiaron el pago de los servicios de la deuda externa y afectaron negativamente los gastos de inversión en infraestructura, en salud y educación, impactaron el crecimiento de la capacidad productiva y del producto de largo plazo.

Existen dos enfoques alternativos sobre el crecimiento económico y el comportamiento del producto de largo plazo. El primero es el enfoque del crecimiento dirigido por la demanda, según el cual, tanto la recesión prolongada de la economía como la política económica impactan en el producto de largo 
plazo. Siguiendo este enfoque se estiman dos modelos de producción de largo plazo para la economía peruana primario-exportadora. Por un lado, está el modelo de Shaikh y Moudud (2004) que se reformuló para captar el cambio técnico determinado por la demanda en el sentido kaldoriano (Kaldor, 1957). Como bien señala Ros (2004 y 2013), el cambio técnico y el crecimiento de la productividad dependen de la acumulación de capital y esta última responde, en el largo plazo, a las variaciones de la demanda. La productividad de los trabajadores aumenta cuanto más y nuevos equipos y máquinas que incorporan desarrollos tecnológicos recientes se integren al proceso de producción; y, cuanto mayor sea la tasa de crecimiento de la productividad, mayor será la tasa de crecimiento de la economía y de la acumulación de capital, generando un proceso de causación circular acumulativa (Ros, 2004 y 2013).

Por otro lado, está el modelo de Thirlwall (1979) que permite estimar tasas teóricas de crecimiento que corresponden al equilibrio de la cuenta corriente (Jiménez, 2009). A partir de estas tasas teóricas se obtiene el producto de largo plazo (en adelante "producto de equilibrio de la balanza de pagos"). Es importante mencionar que, para Clavijo y Ros (2015) el patrón de especialización, factor que revela las condiciones de oferta de la economía, influye en el crecimiento que es lo esencial en Thirlwall. Si un país -dicen-"impulsara un proceso vigoroso de industrialización que reasignara recursos hacia aquellos sectores con mayor dinamismo internacional, generaría cambios en las elasticidades ingreso (de las exportaciones e importaciones) relajando así la restricción externa y acelerando el crecimiento económico" (Clavijo y Ros, 2015, p. 28).

El segundo enfoque es el neoclásico de Solow (1956) y Swan (1956). Es el más conocido y sobre el cual se han desarrollado innumerables trabajos empíricos (Jiménez, 2011). Este enfoque sostiene que el producto de largo plazo de la economía está limitado por factores de oferta, entre los cuales se encuentran el capital y el trabajo. Ambos considerados variables exógenas.

En resumen, el objetivo de este artículo es estimar el producto de largo plazo de la economía peruana a partir de los dos enfoques teóricos mencionados, utilizando una misma técnica econométrica para facilitar su comparación. Como se trata de la estimación del producto de largo plazo, se utiliza la técnica de cointegración pues permite estimar relaciones de largo plazo entre las variables consideradas en los modelos.

El resto del artículo está organizado de la siguiente manera. En la segunda sección se aborda el enfoque del crecimiento dirigido por la demanda y se estima el producto de largo plazo sobre la base de dos modelos: el de Shaikh y Moudud (2004) reformulado con un cambio técnico al de Kaldor y el de 
Thirlwall (1979) de equilibrio de la balanza de pagos. En la tercera sección se presenta el enfoque neoclásico de Solow (1956) y Swan (1956) del crecimiento determinado por factores de oferta y se estima el respectivo producto de largo plazo o producto potencial. Finalmente, se incluye una breve sección de conclusiones en la que se comparan estadísticamente las tres estimaciones.

\section{CRECIMIENTO DE LA CAPACIDAD PRODUCTIVA, CAMBIO TÉCNICO Y PRODUCTIVIDAD DETERMINADOS POR LA DEMANDA}

\section{Capacidad productiva según el enfoque modificado de Shaikh y Moudud}

Según este enfoque, el nivel de producción deseado con un stock de capital dado es lo que constituye el producto de largo plazo, al que los autores denominan capacidad económica productiva, y que co-varía con el stock de capital. No es la máxima producción posible determinada técnicamente para un stock de capital dado; tampoco es el producto potencial definido como el producto de pleno empleo de los factores productivos como sostiene la teoría neoclásica. Es el nivel de producción "deseado" en el sentido de Harrod (1939). El marco teórico adoptado aquí es keynesiano-kaldoriano. Se introduce siguiendo a Kaldor (1957) una ecuación de la tasa de crecimiento del stock de capital como variable endógena determinada por la demanda agregada.

\section{"Capacidad económica productiva", cambio técnico y acumulación de capital determinados por la demanda}

El modelo que incorpora la "capacidad económica productiva", el cambio técnico y la acumulación de capital, se reduce a una identidad y dos ecuaciones de comportamiento. La identidad puede expresarse como sigue:

$$
Y_{t}=\left(\frac{Y_{t}}{Y_{t}^{*}}\right)\left(\frac{Y_{t}^{*}}{K_{t}}\right) K_{t}
$$

donde $Y_{t}$ es el producto; $Y_{t}^{*}$ es el producto de largo plazo (o producto deseado) que Shaikh y Moudud (2004) denominan "capacidad económica", 
y, $K_{t}$ es el stock de capital. El producto y el stock de capital son variables reales observables. Si se identifican cada uno de los dos factores, la identidad anterior adopta la siguiente forma:

$$
Y_{t}=\mu_{t} y_{t}^{*} K_{t}
$$

Donde:

$$
\begin{aligned}
& \mu_{t}=\left(\frac{Y_{t}}{Y_{t}^{*}}\right) \text { es la tasa de utilización de la capacidad productiva, y } \\
& y_{t}^{*}=\left(\frac{Y_{t}^{*}}{K_{t}}\right) \text { es la relación producto deseado-capital }
\end{aligned}
$$

Tomando logaritmos, la identidad se transforma en:

$$
\ln Y_{t}=\ln \mu_{t}+\ln y_{t}^{*}+\ln K_{t}
$$

La tasa de utilización de la capacidad fluctúa alrededor de la unidad. En otras palabras, el desvío $Y_{t}-Y_{t}^{*}$ puede ser menor, mayor o igual a 0 . La tasa de utilización en logaritmos es un error estocástico con media igual a 0 .

$$
\ln \mu_{t}=\ln \frac{Y_{t}}{Y_{t}^{*}}=\ln Y_{t}-\ln Y_{t}^{*}=e_{\mu t}
$$

Siguiendo a Kaldor (1957), la tasa de crecimiento del stock de capital es una variable endógena y responde, a largo plazo, a las variaciones de la demanda. Por lo tanto, esta tasa de crecimiento estará relacionada positivamente con la tasa de crecimiento del producto agregado:

$$
\frac{d K_{t}}{K_{t}}=\delta_{1}+\delta_{2} \frac{d Y_{t}}{Y_{t}}
$$

Donde: $\delta_{1}>0$ y $\delta_{2}>0$. 
Reformulando la ecuación de cambio técnico de Shaikh y Moudud (2004), se sostiene que la variación de la relación producto deseado-capital $(Y * / K)$ responde a un cambio técnico autónomo y a un cambio técnico incorporado que es expresado por la tasa de acumulación de capital. La inversión que incrementa el stock de capital encarna o incorpora progreso tecnológico. Al respecto, el mismo Solow reconoce que:

gran parte del progreso tecnológico, tal vez la mayor parte, podría llegar a la producción real sólo con el uso de equipos de capital nuevos y diferentes. Por lo tanto, la velocidad de la inversión bruta determinará la eficacia de la innovación para aumentar la producción. Una política para aumentar la inversión conduciría, por lo tanto, no sólo a una mayor intensidad de capital, que podría no importar demasiado, sino también a una transferencia más rápida de nueva tecnología a la producción real (1988, pp. 314-315).

La ecuación de cambio técnico tendría, entonces, la forma siguiente:

$$
\frac{d y_{t}^{*}}{y_{t}^{*}}=b_{1}+b_{2} \frac{d K_{t}}{K_{t}}
$$

Donde: $b_{1}>0$ y $b_{2}>0$.

La tasa de acumulación de capital incorpora un cambio técnico que influye positivamente en la productividad "deseada" del capital (coeficiente $b_{2}>0$ ). Pero, además, esta productividad puede aumentar sin relación alguna con la acumulación de capital, es decir, con un cambio técnico autónomo (coeficiente $\left.b_{1}>0\right)$.

Sustituyendo la ecuación (3) en la ecuación (4), se obtiene una relación entre la tasa de variación de la relación producto deseado-capital y la tasa de variación del producto.

$$
\begin{aligned}
& \frac{d y_{t}^{*}}{y_{t}^{*}}=b_{1}+b_{2}\left(\delta_{1}+\delta_{2} \frac{d Y_{t}}{Y_{t}}\right) \\
& \frac{d y_{t}^{*}}{y_{t}^{*}}=\left(b_{1}+b_{2} \delta_{1}\right)+b_{2} \delta_{2} \frac{d Y_{t}}{Y_{t}}
\end{aligned}
$$




$$
\frac{d y_{t}^{*}}{y_{t}^{*}}=\alpha_{1}+\alpha_{2} \frac{d Y_{t}}{Y_{t}}
$$

Donde: $\alpha_{1}=\left(b_{1}+b_{2} \delta_{1}\right)$ y $\alpha_{2}=b_{2} \delta_{2}$.

Según esta ecuación, la "capacidad económica productiva" -que se puede definir también como la relación entre el producto "deseado" de largo plazo de la economía y el stock de capital-, está determinada por la demanda. Integrando esta ecuación y adicionándole un error estocástico se obtiene:

$$
\ln y_{t}^{*}=\alpha_{0}+\alpha_{1} t+\alpha_{2} \ln Y_{t}+e_{y^{*} t}
$$

El coeficiente $\alpha_{1}=\left(b_{1}+b_{2} \delta_{1}\right)$ capta la influencia en el producto de largo plazo, primero, del cambio técnico autónomo y, segundo, del cambio técnico inducido multiplicado por la acumulación de capital autónoma. Un valor negativo de este coeficiente $\alpha_{1}$ podría indicar la presencia, con el paso del tiempo, de un efecto negativo de la tasa de acumulación autónoma que hace que el segundo efecto domine sobre el primero o que el cambio técnico autónomo sea negativo al igual que la tasa de acumulación autónoma, lo que daría lugar a que ambos efectos sean negativos. Formalmente, si $\delta_{1}<0$ y $b_{2} \delta_{1}>b_{1}$ bajo el supuesto de que $b_{1}>0$ y $b_{2}>0$, entonces $\alpha_{1}<0$. Este coeficiente también será negativo si ocurre que $b_{1}<0$ y $\delta_{1}<0$.

Se supone que el coeficiente que capta el cambio técnico inducido $\left(b_{2}\right)$ y el de la acumulación de capital determinado por las variaciones de la demanda $\left(\delta_{2}\right)$ son mayores que 0 . Coeficientes negativos no tendrían sentido económico, pues un aumento de la demanda no podría provocar una desacumulación de capital, ni un aumento de la acumulación de capital puede inducir a una regresión tecnológica. En consecuencia, el parámetro estimado $\alpha_{2}$ será siempre mayor que $0\left(\alpha_{2}=b_{2} \delta_{2}>0\right)$.

Combinando las ecuaciones (1), (2) y (6), se obtiene:

$$
\begin{aligned}
& \ln Y_{t}=e_{\mu t}+\left(\alpha_{0}+\alpha_{1} t+\alpha_{2} \ln Y_{t}+e_{y^{*} t}\right)+\ln K_{t} \\
& \ln Y_{t}=\alpha_{0}+\alpha_{1} t+\alpha_{2} \ln Y_{t}+\left(e_{\mu t}+e_{y_{t}^{*} t}\right)+\ln K_{t} \\
& \left(1-\alpha_{2}\right) \ln Y_{t}=\alpha_{0}+\alpha_{1} t+\ln K_{t}+\left(e_{\mu t}+e_{y_{t}^{*} t}\right)
\end{aligned}
$$




$$
\begin{aligned}
& \ln Y_{t}=\frac{\alpha_{0}}{1-\alpha_{2}}+\frac{\alpha_{1}}{1-\alpha_{2}} t+\frac{1}{1-\alpha_{2}} \ln K_{t}+\frac{\left(e_{\mu t}+e_{y_{t}^{*} t}\right)}{1-\alpha_{2}} \\
& \ln Y_{t}=\varphi_{0}+\varphi_{1} t+\varphi_{2} \ln K_{t}+\varphi_{2} e_{t}
\end{aligned}
$$

Donde: $\varphi_{0}=\frac{\alpha_{0}}{1-\alpha_{2}} ; \varphi_{1}=\frac{\alpha_{1}}{1-\alpha_{2}} ; \varphi_{2}=\frac{1}{1-\alpha_{2}} ; \mathrm{y} e_{t}=\varphi_{2}\left(e_{\mu t}+e_{y_{t}^{\prime \prime} t}\right)$.

La ecuación (7) es diferente a la ecuación (4) de Shaikh y Moudud (2004) incluyendo el término de error. La ecuación indica que los logaritmos del producto $(\mathrm{Y})$ y del stock de capital $(\mathrm{K})$ están cointegrados, con una tendencia determinística posiblemente lineal en los datos reales. Por lo tanto, el valor de largo plazo de la producción real es el producto deseado $Y_{t}^{*}$, que de acuerdo con Shaikh y Moudud (2004) se denomina "capacidad económica productiva".

Nótese que el parámetro $\varphi_{2}$ puede ser menor que 0 o mayor que 0 , pues los parámetros del cambio técnico incorporado $b_{2}$ y de la influencia de la demanda en la acumulación de capital $\delta_{2}$ son ambos positivos. Si el valor estimado del parámetro $\varphi_{2}$ es menor que $0\left(\varphi_{2}<0\right)$, entonces $\alpha_{2}$ será mayor que $1\left(\alpha_{2}>1\right)$. Si $\varphi_{2}>0$ y mayor que la unidad, entonces $\alpha_{2}$ será menor que $1\left(\alpha_{2}<1\right)$. Una vez determinado el producto deseado, es posible encontrar la brecha de producto y la relación capital-producto.

Para estimar la ecuación (7), por el método de cointegración, primero se construyó la serie de stock de capital con precios del año base 2007, actualizando las series de inversión total y de las inversiones en construcción y equipamiento.

\section{El cálculo del stock de capital}

Para construir la nueva serie de stock de capital, se parte de la ecuación de definición del stock de capital para el año $t$ :

$$
K_{t}=I_{t}+\left(1-\delta_{t}\right) K_{t-1}
$$

Partiendo de esta ecuación se obtiene el valor del stock de capital para el año 1955, teniendo como referencia la relación capital-producto estimada por Naciones Unidas (1957). 


$$
\begin{aligned}
& \frac{K_{t}-K_{t-1}}{K_{t-1}} K_{t-1}=I_{t}-\delta_{t} K_{t-1} \\
& \left(g_{w t}+\delta_{t}\right) K_{t-1}=I_{t} \\
& K_{t-1}=\frac{I_{t}}{\left(g_{w t}+\delta_{t}\right)}
\end{aligned}
$$

La tasa de crecimiento $\left(g_{w t}\right)$ es un promedio anual móvil de siete ańos y es la tasa garantizada de Harrod (1939), según la cual el PIB y el stock de capital crecen a una tasa que asegura que la utilización del stock de capital sea la deseada por los capitalistas (Barbosa Filho, 2005; Jiménez, 2011). Por su parte, la tasa de depreciación $\left(\delta_{t}\right)$ sigue la regla lineal considerando una vida útil promedio para las inversiones en equipamiento (maquinaria y equipo) de 20 años y una vida útil promedio para las inversiones en construcción de 60 años. Se calcula como un promedio ponderado por las participaciones de los dos tipos de inversión en el total, las mismas que, como se comprenderá, varían año tras año.

Con la formula anterior y dados los valores de la inversión, de la tasa garantizada y de la tasa de depreciación para año $t$, se calculó el stock de capital de Perú para el ańo 1955. La serie se completa para los años anteriores y posteriores a este último, sustituyendo los datos de inversión y depreciación en la ecuación de definición del stock de capital. ${ }^{1}$ La gráfica 1 muestra las tasas de variación porcentual del stock de capital fijo desde 1950 hasta 2016.

Gráfica 1. Perú: stock de capital fijo (1950-2016) (tasa de crecimiento porcentual)

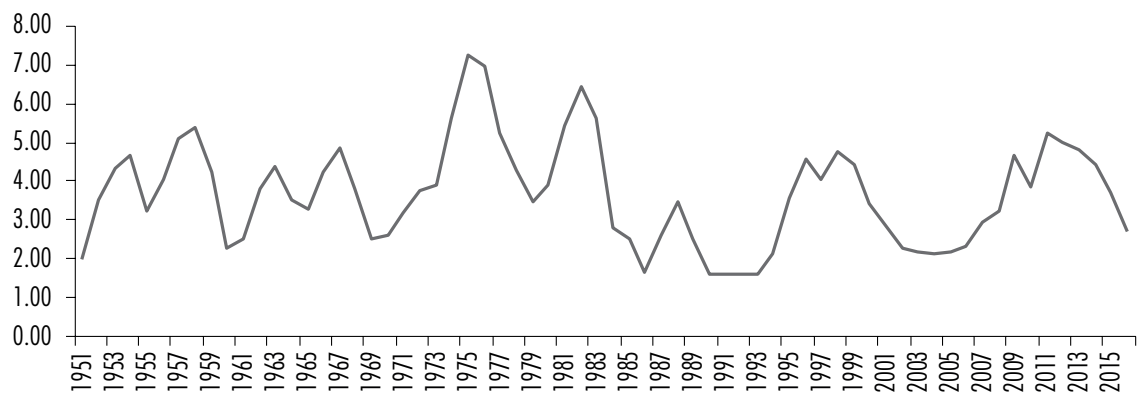

Fuente: estimaciones con base en datos del INEl. Elaboración propia.

1 La serie calculada puede verse en el Apéndice. 
Como estas tasas representan el porcentaje de la inversión neta respecto al stock de capital total, es importante señalar que durante 1950-1983 la tasa de acumulación de capital fue de $4.2 \%$ promedio anual; mientras que durante 1983-2016 fue de sólo 3.1\%. En nueve años de este último periodo (19961999, 2009 y 2010-2014) la tasa de acumulación de capital superó el 4\% anual.

La baja tasa de acumulación en el segundo periodo se refleja en la intensidad de capital (o ratio capital/trabajo) (véase gráfica 2) que, como se sabe, es un indicador de modernización de la economía. Crece sostenidamente de 1950 a 1983, después muestra una tendencia decreciente hasta 2007 cuando alcanza un valor cercano al que se registra en 1975. Podría decirse que el proceso de modernización sostenido que experimentó la economía peruana en el primer periodo estuvo asociado al proceso de industrialización que implicó un incremento significativo de la inversión en maquinaria y equipo. Su participación en la inversión total creció, mientras se redujo la de la inversión en construcción.

Gráfica 2. Perú: relación capital/trabajo (1950-2016) (Miles de soles de 2007 por trabajador)

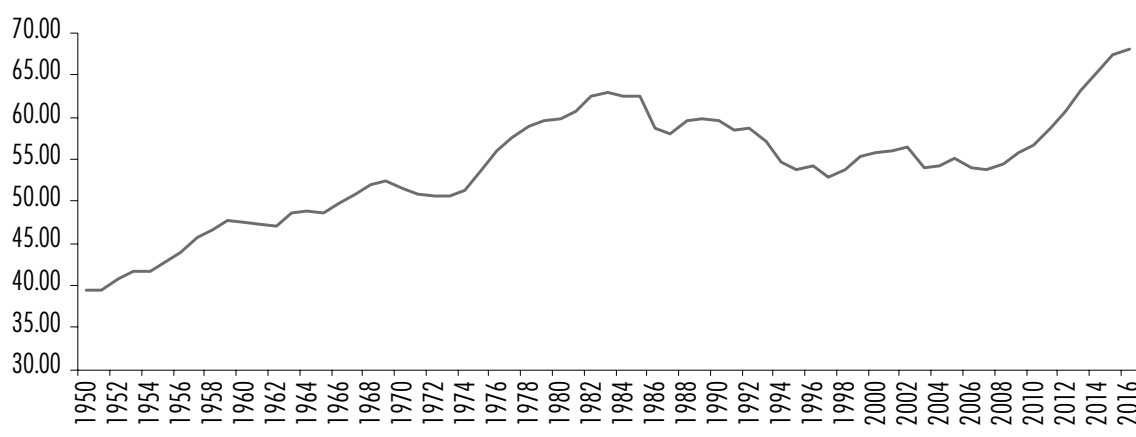

Fuente: INEl. Elaboración propia.

Lo contrario ocurrió en el periodo 1984-2016 (véase gráfica 3). Ya que, además de la crisis de 1980, se presenta un cambio estructural caracterizado por la reprimarización de la economía y una desindustrialización prematura (Jiménez, 2017). De 2008 en adelante, la relación capital/trabajo se recupera, pero en los dos últimos años su crecimiento se desacelera. Es importante mencionar que en el primer periodo hay un crecimiento sostenido de la productividad media del capital, aunque sólo hasta 1974 (véase gráfica 4). Después 
esta productividad cae hasta encontrar su punto más bajo en 1992. Su posterior recuperación se acelera en los años de 2002-2008, para luego estancarse en niveles similares a los registrados en los años de 1982-1983. Un comportamiento parecido registra la productividad media del trabajo, lo que revela que el comportamiento de la productividad en general no estuvo asociado a cambios técnicos importantes, autónomos o inducidos, durante este segundo periodo como se verá más adelante.

Gráfica 3. Perú: inversión en maquinaria y equipo (1950-2016) (porcentaje de participación en el total de inversión)

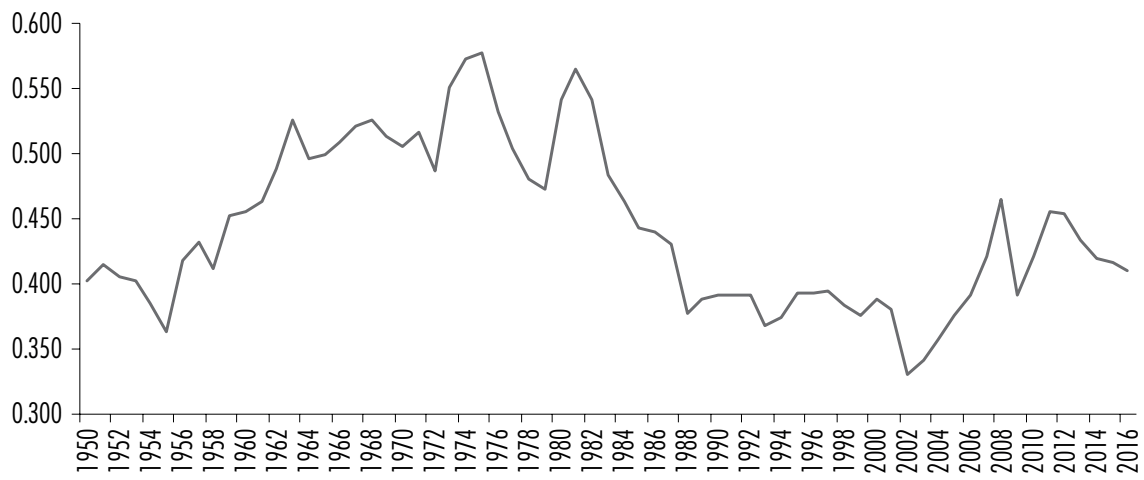

Fuente: INEl. Elaboración propia.

Gráfica 4. Perú: productividad media del stock de capital fiijo (1950-2016) (PIB entre el stock de capital)

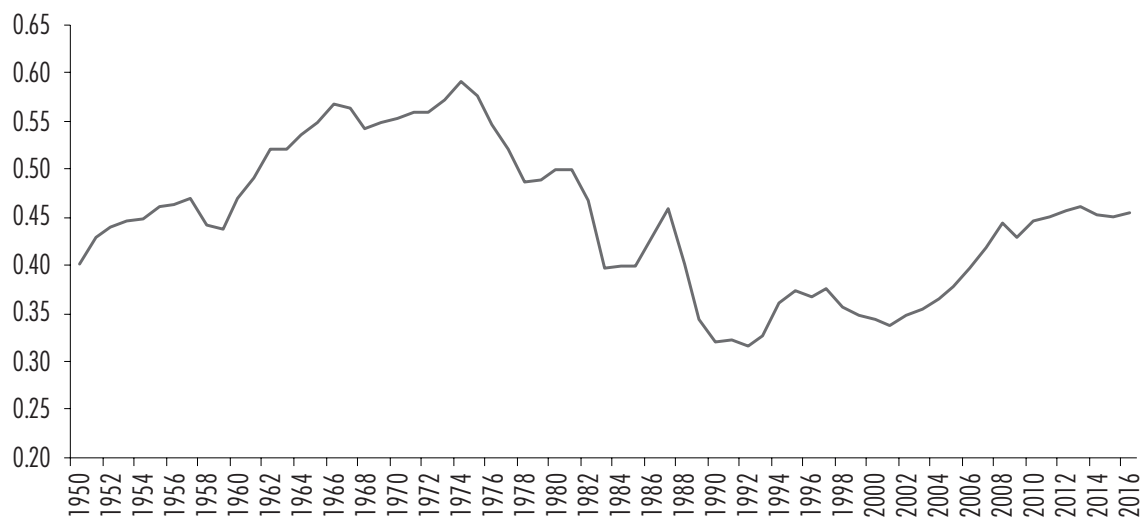

Fuente: INEl. Elaboración propia. 


\section{La relación de cointegración entre el PIB y el stock de capital fijo}

Para comprobar si existe la relación de cointegración, primero tiene que probarse estadísticamente que las series del PIB $(\mathrm{Y})$ y del stock de capital $(\mathrm{K})$ son integradas de orden 1, I(1). Para ello se aplica el test de Dickey-Fuller Aumentado $(\mathrm{ADF})$, incluyendo intercepto y tendencia, sólo intercepto, e intercepto en las primeras diferencias de ambas series. Los rezagos máximos usados fueron cinco y el criterio de selección fue el de Akaike (Akaike Information Criterion, AIC, por sus siglas en inglés).

La hipótesis nula en cada caso es que existe raíz unitaria. Los resultados de los estadísticos $t$ muestran que no se logra rechazar la presencia de raíz unitaria en las series en niveles, ya sea con intercepto solamente o con intercepto y tendencia. El test realizado con las primeras diferencias de las series indica que se rechaza la hipótesis nula de raíz unitaria (véanse cuadros 1 y 2). Por lo tanto, estos resultados indican que ambas series son integradas del orden 1 , en el periodo 1950-2016.

Cuadro 1. Prueba de ráiz unitaria para el PIB de Perú 1950 - 2016

\begin{tabular}{|c|c|c|c|}
\hline Hipótesis nula: & \multicolumn{3}{|c|}{ Log del PIB tiene raíz unitaria } \\
\hline Exógenas: & \multicolumn{3}{|l|}{ Intercepto } \\
\hline Rezagos: & \multicolumn{3}{|c|}{1 con 5 como máximo, AlC } \\
\hline & & Estadístico $t$ & Probabilidad \\
\hline \multicolumn{2}{|c|}{ Test estadístico Dickey-Fuller Aumentado } & -0.623 & 0.857 \\
\hline \multirow[t]{3}{*}{ Valores críticos } & Nivel $1 \%$ & -3.534 & \\
\hline & Nivel 5\% & -2.907 & \\
\hline & Nivel 10\% & -2.591 & \\
\hline Hipótesis nula: & \multicolumn{3}{|c|}{ Log del PIB tiene raíz unitaria } \\
\hline Exógenas: & \multicolumn{3}{|c|}{ Intercepto y tendencia } \\
\hline \multirow[t]{2}{*}{ Rezagos: } & \multicolumn{3}{|c|}{1 con 5 como máximo, AlC } \\
\hline & & Estadistico $t$ & Probabilidad \\
\hline \multicolumn{2}{|c|}{ Test estadístico Dickey-Fuller Aumentado } & -2.011 & 0.584 \\
\hline \multirow[t]{3}{*}{ Valores críticos } & Nivel $1 \%$ & -4.105 & \\
\hline & Nivel 5\% & -3.480 & \\
\hline & Nivel 10\% & -3.168 & \\
\hline
\end{tabular}


Cambio técnico, productividad y estimaciones alternativas del producto de largo plazo

\begin{tabular}{|c|c|c|c|}
\hline Hipótesis nula: & \multicolumn{3}{|c|}{ d(Log del PIB) tiene raíz unitaria } \\
\hline Exógenas: & \multicolumn{3}{|l|}{ Intercepto } \\
\hline Rezagos: & \multicolumn{3}{|c|}{0 con 5 como máximo, AlC } \\
\hline & & Estadístico $†$ & Probabilidad \\
\hline \multicolumn{2}{|c|}{ Test estadístico Dickey-Fuller Aumentado } & -5.132 & 0.000 \\
\hline \multirow[t]{3}{*}{ Valores críticos } & Nivel 1\% & -3.534 & \\
\hline & Nivel 5\% & -2.907 & \\
\hline & Nivel $10 \%$ & -2.591 & \\
\hline
\end{tabular}

Fuente: INEl. Elaboración propia.

Cuadro 2. Prueba de ráiz unitaria para el stock de capital (SK) de Perú 1950 - 2016

\begin{tabular}{|c|c|c|c|}
\hline Hipótesis nula: & \multicolumn{3}{|c|}{ Log del SK total tiene ráiz unitaria } \\
\hline Exógenas: & \multicolumn{3}{|c|}{ Intercepto y tendencia } \\
\hline \multirow[t]{2}{*}{ Rezagos: } & \multicolumn{3}{|c|}{2 con 12 como máximo, AIC } \\
\hline & & Estadistico $t$ & Probabilidad \\
\hline \multicolumn{2}{|c|}{ Test estadístico Dickey-Fuller Aumentado } & -1.073 & 0.722 \\
\hline \multirow[t]{3}{*}{ Valores críticos } & Nivel 1\% & -3.536 & \\
\hline & Nivel 5\% & -2.907 & \\
\hline & Nivel 10\% & -2.591 & \\
\hline Hipótesis nula: & \multicolumn{3}{|c|}{ Log del SK total tiene raíz unitaria } \\
\hline Exógenas: & \multicolumn{3}{|l|}{ Intercepto } \\
\hline \multirow[t]{2}{*}{ Rezagos: } & \multicolumn{3}{|c|}{2 con 5 como máximo, AlC } \\
\hline & & Estadístico $t$ & Probabilidad \\
\hline \multicolumn{2}{|c|}{ Test estadístico Dickey-fuller Aumentado } & -1.562 & 0.797 \\
\hline \multirow[t]{3}{*}{ Valores críticos } & Nivel 1\% & -4.108 & \\
\hline & Nivel 5\% & -3.482 & \\
\hline & Nivel $10 \%$ & -3.169 & \\
\hline Hipótesis nula: & \multicolumn{3}{|c|}{$\mathrm{d}($ Log SK total) tiene ráiz unitaria } \\
\hline Exógenas: & \multicolumn{3}{|l|}{ Intercepto } \\
\hline \multirow[t]{2}{*}{ Rezagos: } & \multicolumn{3}{|c|}{1 con 5 como móximo, AlC } \\
\hline & & Estadístico $t$ & Probabilidad \\
\hline \multicolumn{2}{|c|}{ Test estadístico Dickey-Fuller Aumentado } & -4.489 & 0.001 \\
\hline \multirow[t]{3}{*}{ Valores críticos } & Nivel 1\% & -3.536 & \\
\hline & Nivel 5\% & -2.907 & \\
\hline & Nivel 10\% & -2.591 & \\
\hline
\end{tabular}

Fuente: INEl. Elaboración propia. 
Dado que las series son integradas de orden 1, el siguiente paso es efectuar el test de cointegración de las series. Con este fin se estima la siguiente ecuación con un rezago y sin rezagos.

$$
\ln Y_{t}=\varphi_{0}+\varphi_{1} t+\varphi_{2} \ln K_{t}+e_{t}
$$

En ninguno de los dos casos se encuentra una relación de cointegración, pues los respectivos residuos no siguen un proceso estacionario. La razón que explica este resultado es que las series del PIB y del stock de capital muestran quiebre estructural, lo que implica que el test de cointegración que se debe aplicar debe incorporar dicho quiebre.

El test de Gregory y Hansen (1996a y 1996b) permite evaluar si en la relación de las variables que se supone están cointegradas existe quiebre estructural. Según estos autores, si se ignora la existencia de los quiebres estructurales (en el intercepto, en la tendencia y en el régimen, es decir, en el coeficiente de las variables independientes), se reduce la capacidad del test de cointegración conduciéndonos a la errónea conclusión de que no hay relación de cointegración cuando en realidad sí la hay.

La aplicación del test reporta un valor del estadístico ADF de -5.53 , que está por encima (en valor absoluto) de su valor crítico de -5.5 al 5\%. Lo que indica la existencia de un quiebre estructural en 1984 tomando en cuenta los tres niveles (intercepto, tendencia y cambio de régimen). Los otros dos estadísticos, Za y Zt de Phillips, no indican que exista un quiebre estructural (véase cuadro 3).

Cuadro 3. Test de cointegración con quiebre estructural de Gregory-Hansen

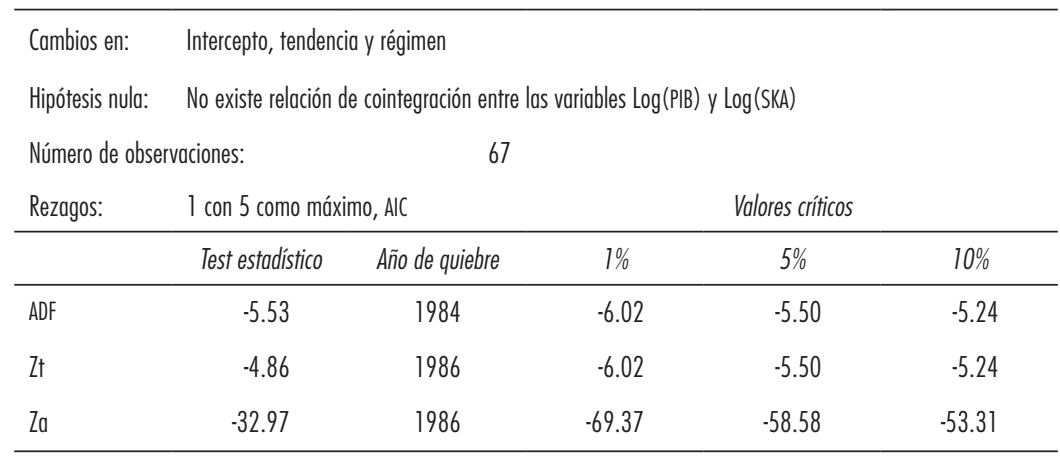

Fuente: INEl. Elaboración propia. 
De acuerdo con esta información se incluirá una variable dummy con valores iguales a 0 para el periodo $1950-1983$ e iguales a 1 para el periodo 1984-2016. La ecuación de cointegración que se someterá a prueba, según el test de Gregory-Hansen, es la siguiente:

$$
\ln Y_{t}=\theta_{0}+\theta_{1} d u m m y+\theta_{2} t+\theta_{3} d u m m y * t+\theta_{4} \ln K_{t}+\theta_{5} d u m m y * \ln K_{t}+e_{t}
$$

donde $Y$ es el PIB; t es la tendencia; $K$ es el stock de capital (sKA); y dummy es la variable ficticia para captar el cambio estructural en el intercepto, en la tendencia, y en el cambio de régimen. Por lo tanto, los parámetros $\varphi_{0}, \varphi_{1}, \mathrm{y}$ $\varphi_{2}$ de la ecuación (7) para los periodos mencionados, serán:

$$
\begin{aligned}
& \varphi_{0}=\left\{\begin{array}{cc}
\theta_{0} & 1950-1983 \\
\theta_{0}+\theta_{1} & 1984-2016 ;
\end{array}\right. \\
& \varphi_{1}=\left\{\begin{array}{cc}
\theta_{2} & 1950-1983 \\
\theta_{2}+\theta_{3} & 1984-2016 ; y
\end{array}\right. \\
& \varphi_{2}=\left\{\begin{array}{cc}
\theta_{4} & 1950-1983 \\
\theta_{4}+\theta_{5} & 1984-2016
\end{array}\right.
\end{aligned}
$$

La estimación se hizo con un rezago de acuerdo al criterio de Akaike y el método utilizado fue el de Mínimos Cuadrados Ordinarios Completamente Modificados (FMOLs, por sus siglas en inglés). Todos los coeficientes estimados de la cointegración son altamente significativos como lo revelan los respectivos valores de los estadísticos ty los p-values (véase cuadro 4).

Cuando se les aplica a los residuos de la ecuación de cointegración estimada, el test de raíz unitaria de ADF -considerando como exógeno el intercepto y con un rezago-, se rechaza la hipótesis nula de existencia de raíz unitaria al $5 \%$ de significancia (véase cuadro 5). Por lo tanto, la estacionariedad de estos residuos muestra que hay una relación de largo plazo entre los logaritmos del PIB y del stock de capital durante el periodo 1950-2016.

Si bien se puede afirmar que las series mantienen una relación de largo plazo, porque los residuos siguen un proceso estacionario, no se puede concluir que esta relación sea estable, es decir, que converja al equilibrio de largo plazo. Para mostrar que es estable, se estima el modelo de corrección de errores mediante Mínimos Cuadrados Ordinarios, considerando como variable de- 
pendiente la diferencia del logaritmo del PIB y como variables independientes las primeras diferencias de los logaritmos del PIB y del stock de capital, más los residuos de la ecuación de cointegración, todos con un rezago.

Cuadro 4. Ecuación de cointegración

\begin{tabular}{lcccc}
\hline Variable dependiente: & Log PIB total & & \\
Método: & Mínimos Cuadrados Ordinarios Completamente Modificados & \\
Muestra: & 1951 - 2016 & & & \\
Observaciones: & 66 & & & \\
\hline Variable & Coeficiente & Error estándar & Estadístico t & Probabilidad \\
\hline Constante & 32.700 & 7.953 & 4.111 & 0.000 \\
dummy & -51.473 & 10.970 & -4.688 & 0.000 \\
tendencia & 0.122 & 0.028 & 4.350 & 0.000 \\
dummy & -0.161 & 0.034 & -4.683 & 0.000 \\
Log(SKA) & -1.911 & 0.692 & -2.762 & 0.007 \\
dummy ${ }^{*}$ Log(SKA) & 4.390 & 0.943 & 4.657 & 0.000 \\
$R^{2}$ ajustado & 0.986 & & & \\
\hline
\end{tabular}

Fuente: INEl. Elaboración propia.

Cuadro 5. Prueba de cointegración

\begin{tabular}{|c|c|c|c|}
\hline Hipótesis nula: & \multicolumn{3}{|c|}{ Residuos con raíz unitaria } \\
\hline Exógenas: & \multicolumn{3}{|l|}{ Intercepto } \\
\hline Rezagos: & \multicolumn{3}{|c|}{1 con 5 como máximo, AlC } \\
\hline & & Estadístico $t$ & Probabilidad \\
\hline \multicolumn{2}{|c|}{ Test estadístico Dickey-Fuller Aumentado } & -4.845 & 0.000 \\
\hline \multirow[t]{3}{*}{ Valores críticos } & Nivel 1\% & -3.540 & \\
\hline & Nivel 5\% & -2.909 & \\
\hline & Nivel 10\% & -2.592 & \\
\hline
\end{tabular}

Fuente: INEl. Elaboración propia. 
El coeficiente del mecanismo de corrección de errores (o coeficiente de los residuos), tiene el signo negativo esperado y su valor igual a -0.207 es estadísticamente distinto de 0 y altamente significativo (véase cuadro 6). Se puede, entonces, afirmar que la relación de cointegración entre el logaritmo del PIB y el logaritmo del stock de capital es estable.

Debido a la significancia de los coeficientes y a que la cointegración es estable estadísticamente, se pueden utilizar los coeficientes estimados de la cointegración con quiebre estructural, para evaluar la pertinencia de las hipótesis de esta investigación.

\begin{tabular}{|c|c|c|c|c|}
\hline \multirow{2}{*}{$\begin{array}{l}\text { Variable dependiente: } \\
\text { Método: }\end{array}$} & \multicolumn{4}{|l|}{$d \log (P \mid B)$} \\
\hline & \multicolumn{4}{|c|}{ Mínimos Cuadrados Ordinarios } \\
\hline Muestra: & \multicolumn{4}{|l|}{$1953-2015$} \\
\hline Observaciones: & \multicolumn{4}{|l|}{65} \\
\hline Variable & Coeficiente & Error estándar & Estadistico $t$ & Probabilidad \\
\hline Constante & 0.0342 & 0.017 & 2.013 & 0.048 \\
\hline$d\left(\log P \mid B_{t-1}\right)$ & 0.535 & 0.118 & 4.533 & 0.000 \\
\hline$d\left(\log S K A n u_{+-1}\right)$ & -0.471 & 0.404 & -1.167 & 0.247 \\
\hline Residuos $_{t-1}$ & -0.207 & 0.074 & -2.783 & 0.007 \\
\hline$R^{2}$ ajustado & 0.316 & & & \\
\hline
\end{tabular}

Fuente: INEl. Elaboración propia.

\section{Cambio técnico y acumulación de capital determinando por la demanda}

Como se recordará, debido al cambio estructural, la constante y los coeficientes hallados mediante la relación de cointegración, tanto del stock de capital como el de la tendencia, no son los mismos para los periodos 1950-1983 y 1984-2016. El coeficiente del stock de capital en el primer periodo tiene un valor negativo de -1.911 , mientras que en el segundo periodo tiene un valor positivo de 2.479 . Asimismo, el coeficiente de la variable tendencia es de 0.122 en el primer periodo y de -0.039 en el segundo. 
A partir de los coeficientes estimados de la ecuación de cointegración con quiebre estructural:

$$
\begin{aligned}
\ln \hat{Y}_{t}= & \hat{\theta}(0)+\hat{\theta}(1) d u m m y+\hat{\theta}(2) t+\hat{\theta}(3) d u m m y * t+\hat{\theta}(4) \ln K_{t} \\
& +\hat{\theta}(5) d u m m y * \ln K_{t} \\
\ln \hat{Y}_{t}= & 32.7-51.473 d u m m y+0.122 t-0.161 d u m m y * t-1.911 \ln K_{t} \\
& +4.39 d u m m y * \ln K_{t}
\end{aligned}
$$

se puede obtener los valores de los coeficientes "alfa" de las ecuaciones (5) y (6), para cada uno de los dos periodos de análisis.

Para el primer periodo (1950-1983) los valores estimados de la constante y de los parámetros de la tendencia y del logaritmo del stock de capital son:

$$
\hat{\theta}(0)=\frac{\hat{\alpha}_{0}}{1-\hat{\alpha}_{2}} ; \hat{\theta}(2)=\frac{\hat{\alpha}_{1}}{1-\hat{\alpha}_{2}} ; \quad y, \quad \hat{\theta}(4)=\frac{1}{1-\hat{\alpha}_{2}}
$$

Por lo tanto, los coeficientes "alpha” $\left(\alpha_{0}, \alpha_{1}\right.$ y $\left.\alpha_{2}\right)$ estimados serán:

$$
\begin{aligned}
& \hat{\alpha}_{0}=\frac{\hat{\theta}(0)}{\hat{\theta}(4)}=\frac{32.7}{-1.911}=-17.111 \\
& \hat{\alpha}_{1}=\frac{\hat{\theta}(2)}{\hat{\theta}(4)}=\frac{0.122}{-1.911}=-0.0638 \\
& \hat{\alpha}_{2}=\frac{\hat{\theta}(4)-1}{\hat{\theta}(4)}=\frac{-1.911-1}{-1.911}=1.523
\end{aligned}
$$

El test de Wald con restricciones (método Delta) revela que todos los coeficientes "alpha" de este primer periodo son estadísticamente diferentes de 0 (véase cuadro 7). En particular, el coeficiente $\alpha_{2}$ es estadísticamente distinto de 1 (mayor que la unidad). Se rechaza la hipótesis nula en cada uno de los cuatro casos. 
Cuadro 7. Test de Wald para el primer periodo (1950 - 1983)

\begin{tabular}{lccccc}
\hline Coeficiente & Valor & $H_{0}$ & Desv. Estándar & t-estadístico & Probabilidad \\
\hline$\alpha_{0}$ & -17.111 & $\alpha_{0}=0$ & 2.033 & -8.414 & 0.000 \\
$\alpha_{1}$ & -0.063 & $\alpha_{1}=0$ & 0.008 & -7.469 & 0.000 \\
$\alpha_{2}$ & 1.523 & $\alpha_{2}=0$ & 0.189 & 8.040 & 0.000 \\
$\alpha_{2-1}$ & 0.523 & $\alpha_{2}=1$ & 0.189 & 2.762 & 0.007 \\
\hline
\end{tabular}

Fuente: elaboración propia.

Para el segundo periodo (1984-2016) los valores estimados de la constante y de los parámetros de la tendencia y del logaritmo del stock de capital, incluyen los coeficientes de las respectivas variables dummy. Estos son:

$$
\hat{\theta}(0)+\hat{\theta}(1)=\frac{\hat{\alpha}_{0}}{1-\hat{\alpha}_{2}} ; \hat{\theta}(2)+\hat{\theta}(3)=\frac{\hat{\alpha}_{1}}{1-\hat{\alpha}_{2}} ; \mathrm{y}, \hat{\theta}(4)+\hat{\theta}(5)=\frac{1}{1-\hat{\alpha}_{2}}
$$

En consecuencia, los coeficientes "alpha" $\left(\alpha_{0}, \alpha_{1} \mathrm{y} \alpha_{2}\right)$ del segundo periodo serán:

$$
\begin{aligned}
& \hat{\alpha}_{0}=\frac{\hat{\theta}(0)+\hat{\theta}(1)}{\hat{\theta}(4)+\hat{\theta}(5)}=\frac{-18.773}{2.479}=-7.573 \\
& \hat{\alpha}_{1}=\frac{\hat{\theta}(2)+\hat{\theta}(3)}{\hat{\theta}(4)+\hat{\theta}(5)}=\frac{-0.039}{2.479}=-0.0157 \\
& \hat{\alpha}_{2}=\frac{[\hat{\theta}(4)+\hat{\theta}(5)]-1}{\hat{\theta}(4)+\hat{\theta}(5)}=\frac{2.479-1}{2.479}=0.597
\end{aligned}
$$

También en este segundo periodo, la aplicación del test de Wald con restricciones (método Delta) conduce a rechazar la hipótesis nula en cada uno de los cuatro casos analizados. 
Félix Jiménez

Cuadro 8. Test de Wald para el segundo periodo (1984 - 2016)

\begin{tabular}{lccccc}
\hline Coeficiente & Valor & $H_{0}$ & Desv. Estándar & t-estadístico & Probabilidad \\
\hline$\alpha_{0}$ & -7.573 & $\alpha_{0}=0$ & 1.098 & -6.894 & 0.000 \\
$\alpha_{1}$ & -0.015 & $\alpha_{1}=0$ & 0.004 & -3.925 & 0.000 \\
$\alpha_{2}$ & 0.597 & $\alpha_{2}=0$ & 0.104 & 5.727 & 0.000 \\
$\alpha_{2 \cdot 1}$ & -0.403 & $\alpha_{2}=1$ & 0.104 & -3.872 & 0.000 \\
\hline
\end{tabular}

Fuente: elaboración propia.

Todos los parámetros "alpha" estimados son significativamente distintos de 0 (véase cuadro 8). Además, el parámetro $\alpha_{2}$ es significativamente menor que la unidad.

Los resultados anteriores permiten diferenciar los efectos de la demanda en el proceso de acumulación de capital y el cambio técnico ocurridos en cada uno de los periodos de análisis. La ecuación que resume ambos efectos para ambos periodos son:

1950-1983:

$$
\begin{aligned}
& \frac{d y_{t}^{*}}{y_{t}^{*}}=\hat{\alpha}_{1}+\hat{\alpha}_{2} \frac{d Y_{t}}{Y_{t}} \\
& \frac{d y_{t}^{*}}{y_{t}^{*}}=-0.063+1.523 \frac{d Y_{t}}{Y_{t}}
\end{aligned}
$$

1984-2016:

$$
\begin{aligned}
\frac{d y_{t}^{*}}{y_{t}^{*}} & =\hat{\alpha}_{1}+\hat{\alpha}_{2} \frac{d Y_{t}}{Y_{t}} \\
\frac{d y_{t}^{*}}{y_{t}^{*}} & =-0.015+0.596 \frac{d Y_{t}}{Y_{t}}
\end{aligned}
$$

donde, como se recordará, $\alpha_{1}=b_{1}+b_{2} \delta_{1}$ y $\alpha_{2}=b_{2} \delta_{2}$ 
Gráfica 5. Perú: productividad media de la PEA Ocupada. (Soles de 2007 por trabajador)

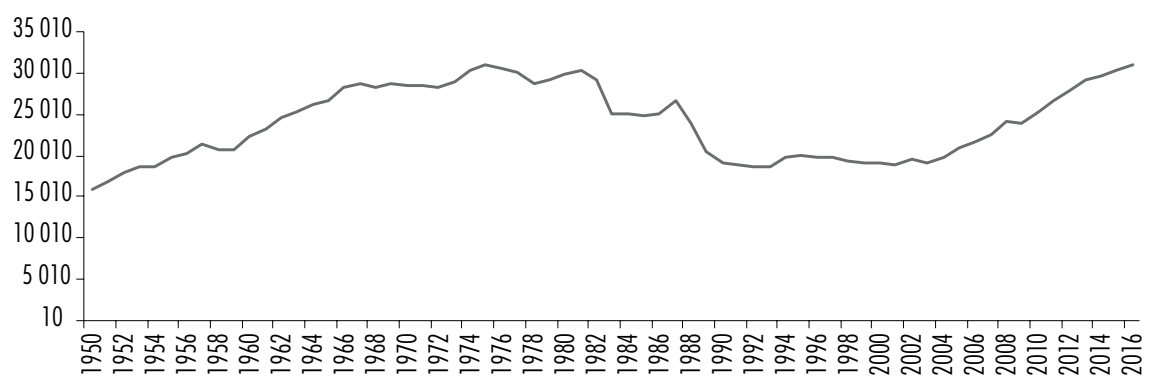

Fuente: INEl. Elaboración propia.

En ambos periodos $\alpha_{2}=b_{2} \delta_{2}>0$, lo que significa que $b_{2}>0$ y $\delta_{2}>0$. El efecto del cambio técnico incorporado $\left(b_{2}\right)$ por el aumento del stock de capital es multiplicado por la influencia de la demanda en la acumulación de capital $\left(\delta_{2}\right)$. Es importante notar que este efecto-multiplicador es mayor en el primer periodo que en el segundo. Recuérdese que, en el primero, a diferencia de lo que ocurrió en el segundo, el capital per cápita crece sostenidamente, al igual que la productividad media del capital.

La productividad media de la Población Económicamente Activa (PEA) Ocupada también crece sostenidamente en el primer periodo y en el segundo disminuye hasta el 2003 y luego se recupera, pero no llega a superar su nivel máximo alcanzado en 1975 (véase gráfica 5).

Por otro lado, el coeficiente $\alpha_{1}=\left(b_{1}+b_{2} \delta_{1}\right)$ incorpora la presencia del cambio técnico autónomo y del cambio técnico inducido multiplicado por la acumulación de capital autónoma. En ambos periodos su valor es negativo. Esto podría indicar la presencia de un efecto negativo de la tasa de acumulación autónoma $\left(\delta_{1}<0\right)$ que hace que $b_{2} \delta_{1}$ sea menor que 0 puesto que $b_{2}$ siempre debe ser positivo. Este efecto estaría dominando a la presencia de cambio técnico autónomo positivo $\left(b_{1}>0\right)$. Pero, puede que la tasa de progreso técnico autónomo sea negativo, $b_{1}<0$, al igual que la tasa de acumulación autónoma, $\delta_{1}<0 ; \mathrm{y}$, en este caso ambos componentes de $\alpha_{1}$ serían negativos $\left(b_{1}<0\right)$ y $\left(b_{2} \delta_{1}<0\right)$. En resumen, todo lo que se puede decir de $\alpha_{1}$ en ambos periodos es que el cambio técnico autónomo y el cambio técnico inducido multiplicado por la tasa autónoma de acumulación, son negativos en ambos periodos. 


\section{El cálculo de la "capacidad económica productiva" $y$ de la brecha del producto}

El producto de largo plazo es el mismo que Shaikh y Moudud (2004) denominan "capacidad económica productiva" y que Harrod (1939) incorpora en la relación producto-capital deseada por los inversionistas. Para el cálculo de este producto se tiene el siguiente procedimiento. En primer lugar, se estima su tasa de crecimiento utilizando la ecuación (7) y los parámetros estimados mediante la regresión de cointegración. En segundo lugar, se identifican los años base en cada uno de los dos periodos (1950-1983 y 1984-2016). Y finalmente, se calcula el producto de largo plazo aplicando a los respectivos años base sus tasas de crecimiento estimadas.

La ecuación (7) llevada al largo plazo con los parámetros estimados, se transforma en:

$$
\frac{d Y_{t}^{*}}{Y_{t}^{*}}=\frac{\hat{\alpha}_{1}}{\left(1-\hat{\alpha}_{2}\right)}+\frac{1}{\left(1-\hat{\alpha}_{2}\right)} \frac{d K_{t}}{K_{t}}
$$

Esta ecuación, con los valores estimados de los parámetros para cada periodo adopta la forma siguiente:

Periodo 1950-1983:

$$
\frac{d Y_{t}^{*}}{Y_{t}^{*}}=0.122-1.911 \frac{d K_{t}}{K_{t}}
$$

Periodo 1984-2016:

$$
\frac{d Y_{t}^{*}}{Y_{t}^{*}}=-0.039+2.479 \frac{d K_{t}}{K_{t}}
$$

Los años base se identificaron con el criterio de la máxima relación producto-capital registrada en cada uno de los periodos. Estos ańos son 1974 y 2013, como se puede observar en la gráfica 4. El pIB registrado en cada uno de estos años es considerado el PIB de largo plazo a partir de cuyos valores se completa la serie para los años restantes mediante las tasas de crecimiento estimadas. El empalme de las series de ambos periodos se efectuó sustituyendo los 
valores del producto de largo plazo encontrados para los años de 1981-1985 por promedios móviles de cinco años centrados en el tercero; procedimiento que modera las tasas de crecimiento de los años de 1983-1984, sin cambiar el sentido de sus variaciones.

El producto de largo plazo (o "capacidad económica productiva") (véase gráfica 6), en la que se revela la reducción del producto de largo plazo originado por las persistentes políticas macroeconómicas contractivas de la demanda. Destaca el último lustro de la década de 1970 en el que se inicia la aplicación de las políticas fiscales y monetarias contractivas y que continúan durante gran parte de la década de 1980. Los años de 1993-1997 son de crecimiento que culminan con la crisis asiática y rusa. La recesión o la contracción de la demanda de los años de 1998-2002 también impacta negativamente en el producto de largo plazo. Hay un nuevo impulso externo al crecimiento durante la década de 2003-2013. La contracción fiscal de los años de 2014-2016 explica la reducción de la pendiente del producto de largo plazo de esos años.

Las líneas discontinuas indican la ruta que habría seguido el producto de largo plazo de no haberse aplicado de modo recurrente políticas de austeridad fiscal y restricción monetaria que contrajeron la demanda y, por lo tanto, desaceleraron el proceso de la acumulación de capital. Los otros pequeños cambios de pendiente en el producto de largo plazo que se aprecian en el periodo 1950-1974 también revelan los años de crisis acompañados por políticas macroeconómicas contractivas.

Gráfica 6. Perú: capacidad económica productiva. (Millones de soles de 2007, en logaritmos)

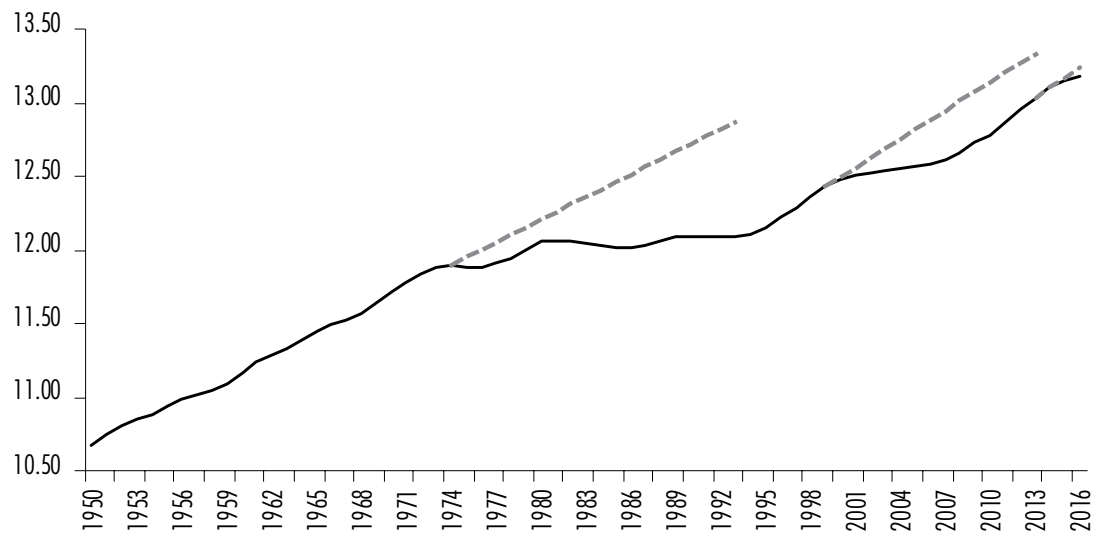

Fuente: estimaciones con base en datos del INEl. Elaboración propia. 
Una vez determinado el producto de largo plazo, se calculó la brecha de producto y la relación producto deseado-capital. La gráfica 7 muestra la brecha estimada como el porcentaje del desvío del producto respecto de su nivel de largo plazo (o "capacidad económica productiva"). Se reproducen claramente las fases de auge y de recesión registrados en los últimos 66 años. Hay siete periodos de auge: 1957, 1962-1967, 1974-1977, 1981-1982, 19851988, 1994-1995 y 2006-2013, y ocho periodos de recesión: 1950-1956, 1958-1961, 1969-1973, 1978-1980, 1983-1984, 1989-1993, 1996-2005 y 2014-2016. Estos periodos, al igual que los años-pico y ańos-valle del ciclo son similares a los encontrados en otros trabajos (Jiménez, 2011).

Gráfica 7. Perú: ciclo económico (1950-2016) (porcentaje)

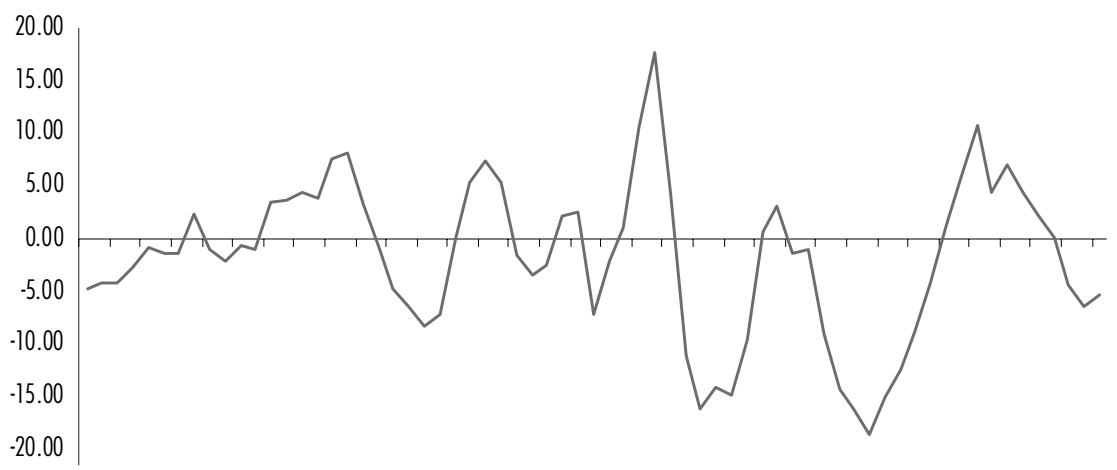

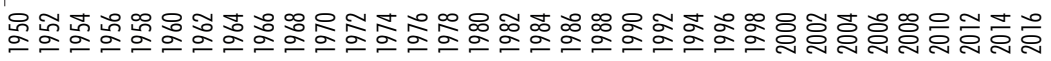

Fuente: estimaciones con base en datos del InEl. Elaboración propia.

Por otro lado, como era de esperarse, la relación producto deseado-capital $\left(Y^{*} / K\right)$, o la productividad media deseada del capital es menor en el segundo periodo 1983-2016 (véase gráfica 8). Esta relación crece hasta 1973, después registra una tendencia decreciente hasta alcanzar un mínimo en 1994. Su recuperación posterior, acentuada en el último periodo de auge por los altos precios de los minerales, permite que se alcance un máximo en 2016, pero que es sólo $81.1 \%$ de su nivel de 1973 y $98.2 \%$ de su nivel de 1981 .

La tasa de crecimiento de la "capacidad económica productiva" se desacelera precisamente en el segundo periodo. Entre 1950 y 1970 crece a una tasa promedio anual de 5.4\%. Si se extiende el periodo en 10 años (1950-1980), la tasa de crecimiento disminuye a $4.73 \%$. Recuérdese que en la segunda mitad de la década de 1970 se aplican políticas de demanda restrictivas que se acentúan en la década de 1980. En los 26 años de neoliberalismo (1990-2016), 
la tasa de crecimiento de la "capacidad económica productiva" alcanzó 4.2\% promedio anual. Si se considera el periodo 1990-2010, la tasa de crecimiento de la capacidad productiva se reduce a $3.5 \%$ promedio anual.

Esta desaceleración tuvo que reducir la capacidad de absorción de la fuerza de trabajo que anualmente se incorpora al mercado. Con una menor "capacidad económica productiva" por persona ocupada, la posibilidad de encontrar empleo -sobre todo, empleo formal-se reduce (véase gráfica 9). En efecto, la "capacidad económica productiva" per cápita crece durante 1950-1970 a la tasa de 3.0\% promedio anual, mientras que durante 1990-2016 lo hace sólo a la tasa de $1.4 \%$. Esta tasa se explica por el alto crecimiento económico de los últimos seis años que se trunca con la crisis internacional y la caída de las inversiones.

Gráfica 8. Perú: relación producto deseado entre el stock de capital

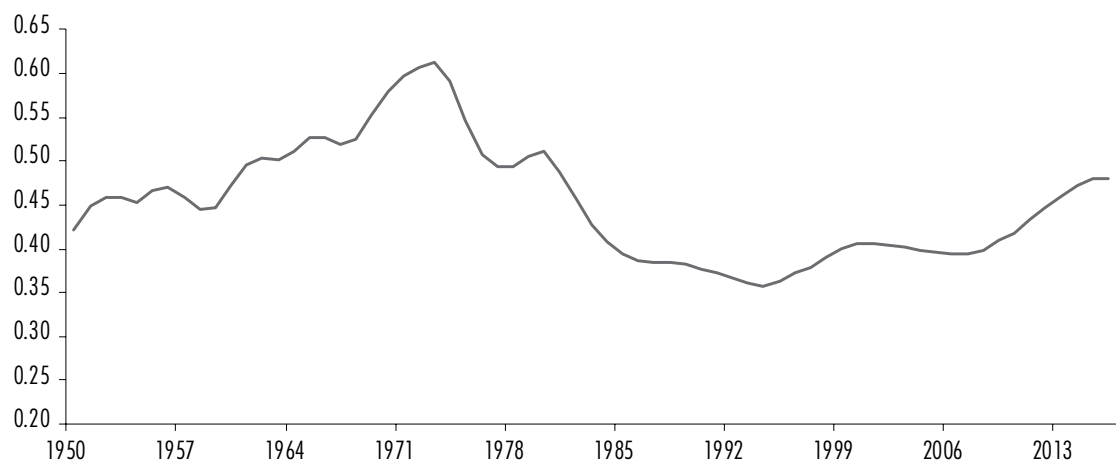

Fuente: estimaciones con base en datos del INEl. Elaboración propia.

Gráfica 9. Capacidad económica productiva entre la PeA Ocupada. (Soles de 2007 por trabajador)

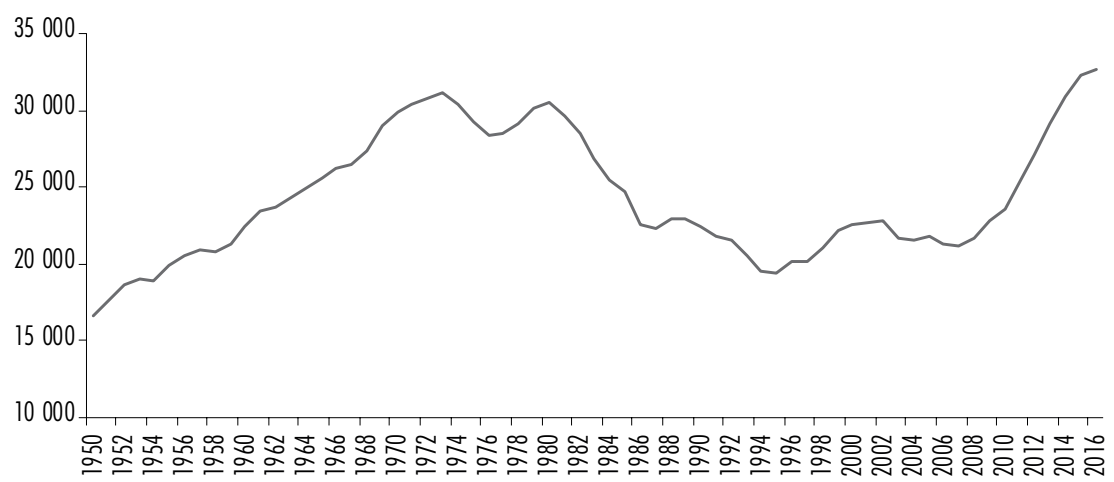

Fuente: estimaciones con base en datos del INEl. Elaboración propia. 
El impacto negativo de las políticas restrictivas de demanda que se aplicaron desde la segunda mitad de la década de 1970, en adelante redujo la capacidad productiva de manera sistemática haciéndola retroceder hasta alcanzar un nivel mínimo en 1995 y que equivale a 97.5\% del registrado en 1955 . Después de 1995 crece lentamente hasta el 2010 a la tasa de 1.3\% promedio anual. Su aceleración posterior permite que la capacidad productiva se recupere hasta superar en 2016 al nivel máximo de 1973 en 4.9\%.

\section{El producto de equilibrio de la balanza de pagos de Thirlwall}

Las reformas y políticas neoliberales aplicadas en los últimos 30 años transformaron la estructura productiva, haciéndola menos agrícola y manufacturera, con un marcado sesgo primario exportador, y más productora de servicios de baja productividad donde se concentra la mano de obra no calificada y de bajos ingresos. Este cambio estructural redujo la participación de la producción para el mercado interno, al mismo tiempo que acrecentó la penetración de las importaciones. La economía se hizo entonces más dependiente de importaciones y de la demanda externa para sostener su crecimiento en el largo plazo. ${ }^{2}$ En consecuencia, podría afirmarse que el comportamiento del producto de equilibrio de la balanza de pagos habría sido influido por la demanda externa a través de las exportaciones y los ciclos de los términos del intercambio (Thirlwall, 1979). Según este autor, la economía no puede crecer en el largo plazo por encima de la tasa de crecimiento de equilibrio de la balanza de pagos. Se supone que el financiamiento externo de los déficits en la cuenta corriente de la balanza de pagos no puede ser indefinido.

\section{El modelo general de equilibrio de la balanza de pagos}

Para corroborar esta hipótesis se parte del modelo de Harrod-Thirlwall, conocido como la Ley de Thrilwall (McCombie y Thrilwall, 1994; Thirlwall y Hussain, 1982; Thirlwall, 2003), para el periodo 1950-2016. El modelo contiene una condición de equilibrio de la cuenta corriente de la balanza de pagos (en

2 Un análisis de las restricciones al crecimiento de la economía peruana durante 1950-1981 puede verse en Jiménez (1984) y la aplicación del modelo de Thirlwall para el periodo 1950-1985 en Jiménez (1988). 
adelante "equilibrio de la balanza de pagos") y las ecuaciones de demanda de exportaciones y de importaciones:

$$
\begin{aligned}
& P_{d t} X_{t}=P_{f t} M_{t} \\
& M_{t}=\left(\frac{P_{f t}}{P_{d t}}\right)^{\eta}\left(Y_{t}\right)^{\pi} \\
& X_{t}=\left(\frac{P_{d t}}{P_{f t}}\right)^{\delta}\left(Y_{t} *\right)^{\theta}
\end{aligned}
$$

donde $P_{d t}$ y $P_{f t}$ son los precios de las exportaciones y de las importaciones, ambos expresados en moneda doméstica, y $X_{t}$ y $M_{t}$ son las cantidades de exportaciones e importaciones a precios constantes. En las ecuaciones de demanda cuya forma es estándar, $Y_{t}$ y $Y_{t}^{*}$ son los ingresos doméstico y del resto del mundo, y los exponentes $\eta_{\text {y }} \delta$ son las elasticidades precio de la demanda de importaciones y exportaciones, mientras que $\pi$ y $\theta$ son las elasticidades ingreso de las importaciones y exportaciones, respectivamente.

Tomando logaritmos y diferenciando las ecuaciones de demanda se obtiene:

$$
\begin{aligned}
& \dot{m}_{t}=\eta\left(\dot{p}_{f t}-\dot{p}_{d t}\right)+\pi \dot{y}_{t} \\
& \dot{x}_{t}=-\delta\left(\dot{p}_{f t}-\dot{p}_{d t}\right)+\theta \dot{y}_{t}^{*}
\end{aligned}
$$

Aplicando el mismo procedimiento a la ecuación de equilibrio de la balanza de pagos se obtiene:

$$
\begin{aligned}
& \dot{p}_{d t}+\dot{x}_{t}=\dot{p}_{f t}+\dot{m}_{t} \\
& \dot{p}_{d t}-\delta\left(\dot{p}_{f t}-\dot{p}_{d t}\right)+\theta \dot{y}_{t}^{*}=\dot{p}_{f t}+\eta\left(\dot{p}_{f t}-\dot{p}_{d t}\right)+\pi \dot{y}_{t} \\
& \pi \dot{y}_{t}=(1+\delta+\eta)\left(\dot{p}_{d t}-\dot{p}_{f t}\right)+\theta \dot{y}_{t}^{*}
\end{aligned}
$$

Como:

$$
\dot{x}_{t}-\delta\left(\dot{p}_{d t}-\dot{p}_{f t}\right)=\theta \dot{y}_{t}^{*}
$$


Reemplazando en la ecuación anterior, la tasa de crecimiento de equilibrio de la balanza de pagos es igual a:

$$
\begin{aligned}
& \pi \dot{y}_{t}=(1+\delta+\eta)\left(\dot{p}_{d t}-\dot{p}_{f t}\right)+\dot{x}_{t}-\delta\left(\dot{p}_{d t}-\dot{p}_{f t}\right) \\
& \dot{y}_{t}=\frac{(1+\eta)\left(\dot{p}_{d t}-\dot{p}_{f t}\right)+\dot{x}_{t}}{\pi}
\end{aligned}
$$

\section{Producto de equilibrio de la balanza de pagos}

Se supone, siguiendo a Thirlwall y Hussain (1982), que los términos de intercambio no afectan el producto en el largo plazo, lo que hace que la tasa teórica de crecimiento de equilibrio de la balanza de pagos se reduzca a: ${ }^{3}$

$$
\dot{y}_{t}=\frac{\dot{x}_{t}}{\pi}
$$

Esta ecuación es conocida como la Ley de Thirlwall, donde $1 / \pi$ es el multiplicador del comercio exterior de Harrod. Las exportaciones son concebidas como el determinante más importante del crecimiento de largo plazo. De acuerdo con Thirlwall (2003, p. 84):

Si no hay ingresos de exportación para financiar el contenido importado de los otros componentes del gasto, entonces la demanda deberá ser restringida. En este aspecto, las exportaciones son de gran significado si el equilibrio de la cuenta corriente de la balanza de pagos es un requerimiento a largo plazo. Esto significa que las exportaciones tienen no sólo un efecto directo sobre la demanda, sino que, además, tienen un efecto indirecto al permitir que todos los otros componentes de la demanda crezcan más rápido que en cualquier otro caso.

3 Los precios relativos no afectan el producto porque se supone que están estables en el largo plazo, de modo que se cumple la paridad del poder adquisitivo (Thirlwall, 1979). 
La Ley de Thirlwall expresa, entonces, una relación de largo plazo; por lo tanto, para estimar esta relación mediante la técnica de cointegración ${ }^{4}$ habrá de expresarse en términos de los logaritmos del piв $\left(\ln Y_{t}\right)$ y de las exportaciones reales de bienes y servicios $\left(\ln X_{t}\right)$.

Integrando la ecuación (12) y agregándole una perturbación aleatoria se obtiene:

$$
\begin{aligned}
& \ln Y_{t}=\beta_{0}+\beta_{1} \ln X_{t}+e_{t} \\
& \text { donde: } \beta_{1}=\frac{1}{\pi}
\end{aligned}
$$

\section{El test raiz unitaria \\ y la cointegración}

Esta ecuación es la que se va a estimar mediante el método de cointegración. El logaritmo del PIB tiene raíz unitaria, por lo tanto, sólo se tiene que evaluar si el logaritmo de la serie de exportaciones también es integrada de orden 1.

El resultado del test de raíz unitaria de ADF considerando intercepto, e intercepto y tendencia en conjunto, indica que no se puede rechazar la hipótesis nula de que el logaritmo de las exportaciones tiene raíz unitaria (véase cuadro 9). Cuando se aplica el test a las primeras diferencias del logaritmo de las exportaciones, utilizando sólo el intercepto, se confirma su estacionariedad.

Por lo tanto, dado que ambas series -el logaritmo del PIB y el de las exportaciones-, son integradas de orden 1 , se puede proceder a estimar la ecuación (13) mediante el método de cointegración. Sin embargo, previamente se tiene que realizar los test de cambio estructural para identificar estadísticamente si hay quiebres resultantes de los cambios en los regímenes de política y del entorno internacional que afectaron el comportamiento de las exportaciones.

4 Para el uso de esta técnica en la estimación del modelo de Thirlwall, véase Atesoglu (1997) y MorenoBrid (1998). 
Félix Jiménez

Cuadro 9. Prueba de raíz unitaria para las exportaciones totales (Xbss) de Perú $1950-2016$

\begin{tabular}{|c|c|c|c|}
\hline Hipótesis nula: & \multicolumn{3}{|c|}{ Log de Xbss total tiene ráiz unitaria } \\
\hline Exógenas: & \multicolumn{3}{|l|}{ Intercepto } \\
\hline \multirow[t]{2}{*}{ Rezagos: } & \multicolumn{3}{|c|}{0 con 5 como máximo, AlC } \\
\hline & & Estadístico $t$ & Probabilidad \\
\hline \multicolumn{2}{|c|}{ Test estadístico Dickey-Fuller Aumentado } & -0.766 & 0.822 \\
\hline \multirow[t]{3}{*}{ Valores críticos } & Nivel 1\% & -3.533 & \\
\hline & Nivel 5\% & -2.906 & \\
\hline & Nivel 10\% & -2.591 & \\
\hline Hipótesis nula: & \multicolumn{3}{|c|}{ Log del Xbss total tiene ráiz unitaria } \\
\hline Exógenas: & \multicolumn{3}{|c|}{ Intercepto y tendencia } \\
\hline \multirow[t]{2}{*}{ Rezagos: } & \multicolumn{3}{|c|}{0 con 5 como máximo, AlC } \\
\hline & & Estadístico $t$ & Probabilidad \\
\hline \multicolumn{2}{|c|}{ Test estadístico Dickey-Fuller Aumentado } & -1.679 & 0.750 \\
\hline \multirow[t]{3}{*}{ Valores críticos } & Nivel 1\% & -4.103 & \\
\hline & Nivel 5\% & -3.479 & \\
\hline & Nivel 10\% & -3.167 & \\
\hline Hipótesis nula: & \multicolumn{3}{|c|}{ d(Log Xbss total) tiene ráiz unitaria } \\
\hline Exógenas: & \multicolumn{3}{|l|}{ Intercepto } \\
\hline \multirow[t]{2}{*}{ Rezagos: } & \multicolumn{3}{|c|}{0 con 5 como máximo, AIC } \\
\hline & & Estadistico $t$ & Probabilidad \\
\hline \multicolumn{2}{|c|}{ Test estadístico Dickey-Fuller Aumentado } & -7.740 & 0.000 \\
\hline \multirow[t]{3}{*}{ Valores críticos } & Nivel 1\% & -3.535 & \\
\hline & Nivel 5\% & -2.907 & \\
\hline & Nivel 10\% & -2.591 & \\
\hline
\end{tabular}

Fuente: estimaciones con base en datos del BCRP. Elaboración propia.

El test de cointegración con quiebre estructural de Gregory y Hansen -que permite analizar quiebres en la relación conjunta de los logaritmos del PIB y de las exportaciones- reportó un cambio estructural en 1990, pero que no es estadísticamente significativo (véase cuadro 10). Por lo tanto, se optó por evaluar sólo la serie de exportaciones mediante el test de Zivot y Andrews. 
Cuadro 10. Test de cointegración con quiebre estructural de Gregory-Hansen

\begin{tabular}{|c|c|c|c|c|c|}
\hline $\begin{array}{l}\text { Cambios en: } \\
\text { Hipótesis nula: }\end{array}$ & \multicolumn{5}{|c|}{ Intercepto, tendencia y régimen } \\
\hline \multicolumn{6}{|c|}{ Número de observaciones: } \\
\hline \multirow[t]{2}{*}{ Rezagos: } & \multicolumn{2}{|c|}{1 con 5 como máximo, AlC } & \multicolumn{3}{|c|}{ Valores críticos } \\
\hline & Test Estadístico & Año de quiebre & $1 \%$ & $5 \%$ & $10 \%$ \\
\hline ADF & -5.17 & 1990 & -6.02 & -5.5 & -5.24 \\
\hline Z† & -4.93 & 1989 & -6.02 & -5.5 & -5.24 \\
\hline Za & -33.97 & 1989 & -69.37 & -58.58 & -53.31 \\
\hline
\end{tabular}

Fuente: estimaciones con base en datos del BCRP. Elaboración propia.

$\mathrm{Al}$ aplicar el test de Zivot y Andrews sólo con el intercepto, el quiebre estructural para las exportaciones se sitúa en el año 1980 (véase cuadro 11). Lo que puede explicarse por la caída notable en su tasa de crecimiento después de 1979, que da lugar al estancamiento de sus niveles. El valor real que las exportaciones alcanzan en 1979 sólo se llega a superar en 1994.

El estancamiento de las exportaciones en el periodo 1980-1993 (véase gráfica 9) revela cambios en el intercepto durante los años de 1950-2016. Podría decirse que hay un intercepto para el periodo 1950-1979, otro para 1980-1993 y el último para 1994-2016. Cuando se toma en cuenta únicamente la tendencia, el test reporta un quiebre estructural en 1991, que es el año en que las exportaciones empiezan a recuperarse. En el periodo anterior 1980-1990, las exportaciones decrecieron a la tasa de $-1.4 \%$ promedio anual. Después, entre 1990 y 2008, crecen sostenidamente a la tasa de $8.1 \%$ promedio anual. En los ańos 2009, 2013 y 2014 decrecen $(-0.8,-1.3$ y $-0.9 \%$, respectivamente); pero en el conjunto del periodo 2008-2016, las exportaciones crecen a la tasa de $3.0 \%$ promedio anual. Finalmente, al aplicarse el test considerando intercepto y tendencia, se registra un quiebre estructural en 1983 (véanse cuadro 11 y gráfica 10). 
Félix Jiménez

Cuadro 11. Test de ráiz unitaria de Zivot y Andrews para las exportaciones

\begin{tabular}{|c|c|c|}
\hline Muestra: & \multicolumn{2}{|c|}{1950 - 2016} \\
\hline Observaciones: & \multicolumn{2}{|l|}{67} \\
\hline Hipótesis nula: & \multicolumn{2}{|c|}{ Log(Xbss) tiene ráiz unitaria con un quiebre estructural en el intercepto } \\
\hline Rezagos: & \multicolumn{2}{|c|}{0 (con 4 como máximos) } \\
\hline Año de quiebre: & \multicolumn{2}{|l|}{1980} \\
\hline \multicolumn{3}{|r|}{ testadístico } \\
\hline \multicolumn{2}{|c|}{ Test estadístico de Zivot-Andrews } & -3.202 \\
\hline \multicolumn{2}{|l|}{ valor crítico al $1 \%$} & -5.34 \\
\hline \multicolumn{2}{|l|}{ valor crítico al $5 \%$} & -4.93 \\
\hline \multicolumn{2}{|l|}{ valor crítico al 10\% } & -4.58 \\
\hline Muestra: & \multicolumn{2}{|c|}{$1950-2016$} \\
\hline Observaciones: & \multicolumn{2}{|l|}{67} \\
\hline Hipótesis nula: & \multicolumn{2}{|c|}{ Log(Xbss) tiene raíz unitaria con un quiebre estructural en la tendencia } \\
\hline Rezagos & \multicolumn{2}{|c|}{0 (con 4 como máximos) } \\
\hline \multirow[t]{2}{*}{ Año de quiebre } & \multicolumn{2}{|l|}{1991} \\
\hline & \multicolumn{2}{|r|}{ testadístico } \\
\hline \multicolumn{2}{|c|}{ Test estadístico de Zivot-Andrews } & -2.424 \\
\hline \multicolumn{2}{|c|}{ valor crítico al $1 \%$} & -4.8 \\
\hline \multicolumn{2}{|l|}{ valor crítico al $5 \%$} & -4.42 \\
\hline \multicolumn{2}{|l|}{ valor crítico al 10\% } & -4.11 \\
\hline Muestra: & \multicolumn{2}{|c|}{$1950-2016$} \\
\hline Observaciones: & \multicolumn{2}{|l|}{67} \\
\hline Hipótesis nula: & \multicolumn{2}{|c|}{ Log(Xbss) tiene raíz unitaria con un quiebre estructural en el intercepto y tendencia } \\
\hline Rezagos & \multicolumn{2}{|c|}{0 (con 4 como máximos) } \\
\hline \multirow[t]{2}{*}{ Año de quiebre } & 1983 & \\
\hline & & t-estadístico \\
\hline \multicolumn{2}{|c|}{ Test estadístico de Zivot-Andrews } & -3.058 \\
\hline \multicolumn{2}{|l|}{ valor crítico al $1 \%$} & -5.57 \\
\hline \multicolumn{2}{|l|}{ valor crítico al 5\% } & -5.08 \\
\hline \multicolumn{2}{|l|}{ valor crítico al 10\% } & -4.82 \\
\hline
\end{tabular}

Fuente: estimaciones con base en datos del BCRP. Elaboración propia. 
Gráfica 10. Perú: PIB y exportaciones de bienes y servicios

(Millones de soles de 2007, en logaritmos)

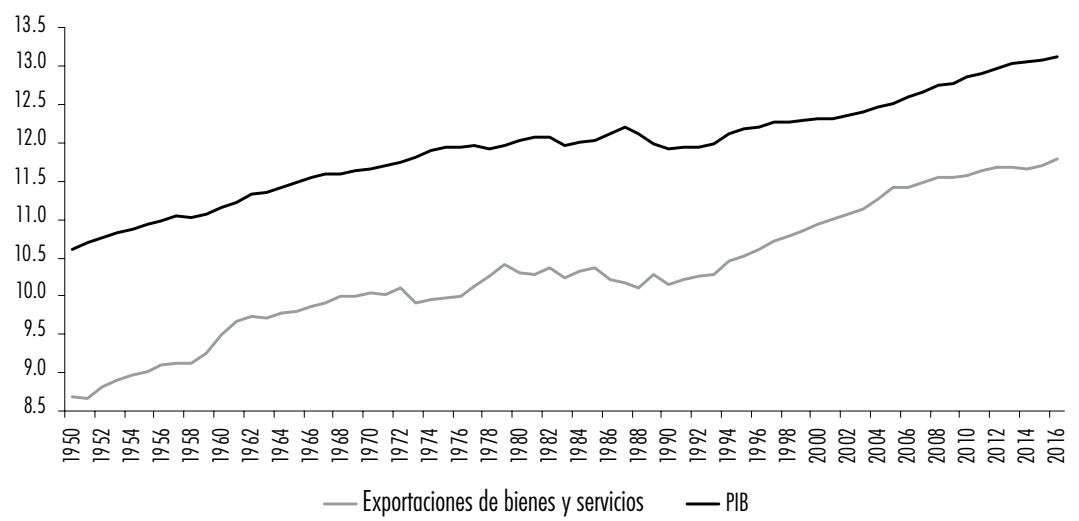

Fuente: estimaciones con base en datos del BCRP. Elaboración propia.

Tomando en cuenta los resultados del análisis del cambio estructural, para estimar la ecuación (13) mediante el método de cointegración, se incorpora una variable dummy (dummy84) con el valor de 1 desde 1984 en adelante, la misma que multiplicará al logaritmo de las exportaciones de bienes y servicios. La idea es que esta dummy capte el cambio de régimen debido a la existencia de dos periodos claramente distintos en el comportamiento de las exportaciones de bienes y servicios. Asimismo, se incluye otra variable dummy (dummy91) con el valor de 1 para el periodo 1991-2016 con el propósito de captar el cambio en la tendencia en la relación entre las series de PIB y de exportaciones.

Los resultados de la estimación de la ecuación de cointegración pueden verse en el cuadro 12. La ecuación incluye una constante, la tendencia, la tendencia multiplicada por la dummy91, el logaritmo de las exportaciones de bienes y servicios y, finalmente, esta última variable multiplicada por la dummy84. El método de estimación utilizado fue el de FMOLs.

Todos los coeficientes estimados son estadísticamente significativos. El coeficiente de la tendencia tiene un valor de 0.033 para el periodo $1950-1990$, y un valor de 0.029 para el de 1991-2016. De otro lado, el coeficiente del logaritmo de las exportaciones tiene un valor de 0.297 para el periodo 1950-1983 y de 0.266 para el de 1984-2016. 
Félix Jiménez

Cuadro 12. Ecuación de cointegración

\begin{tabular}{lcccc}
\hline Variable dependiente: & Log PIB total & & \\
Método: & Mínimos Cuadrados Ordinarios Completamente Modificados & \\
Muestra: & 1951 - 2016 & & \\
Observaciones: & 66 & & & \\
\hline Variable & Coeficiente & Error estándar & Estadísticot & Probabilidad \\
\hline Constante & 8.062 & 0.526 & 15.342 & 0.000 \\
tendencia & 0.033 & 0.003 & 9.610 & 0.000 \\
tendencia^dummy91 & -0.004 & 0.001 & -5.744 & 0.000 \\
Log(Xbss) & 0.297 & 0.059 & 5.000 & 0.000 \\
Log(Xbss)*dummy84 & -0.031 & 0.005 & -6.067 & 0.000 \\
$R^{2}$ ajustado & 0.984 & & & \\
\hline
\end{tabular}

Fuente: estimaciones con base en datos del BCRP. Elaboración propia.

La existencia de cointegración entre los logaritmos del pIB y de las exportaciones se confirma con el test de estacionariedad de los residuos. El test de raíz unitaria de ADF, aplicado a los residuos con intercepto y un rezago, revela que son estacionarios (véase cuadro 13). El valor estimado del estadístico $t$ igual a -3.996, es mayor, en valor absoluto, que su valor crítico (-3.537) al $1 \%$.

\begin{tabular}{|c|c|c|c|}
\hline Hipótesis nula: & \multicolumn{3}{|c|}{ Residuos con raíz unitaria } \\
\hline Exógenas: & \multicolumn{3}{|l|}{ Intercepto } \\
\hline Rezagos: & \multicolumn{3}{|c|}{1 con 5 como máximo, AlC } \\
\hline & & Estadístico $t$ & Probabilidad \\
\hline \multicolumn{2}{|c|}{ Test estadístico Dickey-Fuller Aumentado } & -3.996 & 0.003 \\
\hline \multirow[t]{3}{*}{ Valores críticos } & Nivel 1\% & -3.537 & \\
\hline & Nivel 5\% & -2.908 & \\
\hline & Nivel 10\% & -2.591 & \\
\hline
\end{tabular}

Fuente: estimaciones con base en datos del BCRP. Elaboración propia. 
Para corroborar si esta relación de cointegración es estable, se estima el modelo de corrección errores, incorporando como variable dependiente la primera diferencia del logaritmo del PIB y como variables independientes los residuos de la ecuación de cointegración con rezago de un periodo, la primera diferencia del logaritmo del PIB rezagado un periodo y del logaritmo de las exportaciones de bienes y servicios (Troncoso-Valverde, 2004). El coeficiente correspondiente al mecanismo de corrección de errores es negativo y estadísticamente significativo, con valor de -0.254 (véase cuadro 14).

\begin{tabular}{|c|c|c|c|c|}
\hline Variable dependiente: & \multicolumn{4}{|l|}{$\mathrm{d} \log (\mathrm{PIB})$} \\
\hline Método: & \multicolumn{4}{|c|}{ Mínimos Cuadrados Ordinarios } \\
\hline Muestra: & \multicolumn{4}{|l|}{$1952-2015$} \\
\hline Observaciones: & \multicolumn{4}{|l|}{65} \\
\hline Variable & Coeficiente & Error estándar & Estadístico $t$ & Probabilidad \\
\hline Constante & 0.018 & 0.007 & 2.668 & 0.010 \\
\hline$d\left(\log P \mid B_{+1}\right)$ & 0.584 & 0.114 & 5.106 & 0.000 \\
\hline $\mathrm{d}\left(\right.$ LogXbss $\left._{\mathrm{H}-1}\right)$ & -0.079 & 0.064 & -1.132 & 0.262 \\
\hline Residuos $+1_{-1}$ & -0.254 & 0.070 & -3.644 & 0.001 \\
\hline$R^{2}$ ajustado & 0.326 & & & \\
\hline
\end{tabular}

Fuente: estimaciones con base en datos del BCRP. Elaboración propia.

\section{El producto de equilibrio de la balanza de pagos y la brecha del producto}

La ecuación de cointegración estimada reporta coeficientes de las exportaciones distintos de 0 que permiten obtener dos elasticidades de importaciones a PIB: 3.3 para el periodo $1950-1983$ y 3.7 para el periodo 1984-2016. Pero, según el test de Wald, no son significativamente diferentes. Los coeficientes estimados no captan el incremento de la elasticidad de las importaciones respecto a la demanda interna ocurrido en los últimos 26 ańos: la liberalización comercial y la apreciación cambiaria incrementaron notablemente la penetración de las importaciones en el mercado doméstico. Además, siguiendo a Ros (2009), la apreciación del tipo de cambio real habría estimulado la inversión fija al reducir los precios relativos de la maquinaria y equipo importados, pero esta reducción de precios favorece a las actividades de producción de bienes 
no transables, reduce la rentabilidad en las actividades de bienes transables y, por lo tanto, afecta negativamente la acumulación de capital en este último tipo de actividades.

Por otro lado, puede decirse que la generación de una misma tasa de crecimiento del producto que corresponda al equilibrio de la balanza de pagos, requiere, en el segundo periodo, una tasa de crecimiento mayor de exportaciones que el requerido en el primer periodo. Asimismo, se puede decir que una misma tasa de crecimiento de la producción aumenta más las importaciones en el segundo periodo que en el primero. Las políticas neoliberales habrían hecho más vulnerable el sector externo de la economía peruana, aunque el boom primario exportador de los ańos 2003-2013, por los altos precios de las materias primas, disipó temporalmente este efecto.

Con los coeficientes estimados se obtuvieron las tasas de crecimiento teóricas que corresponden al equilibrio de la cuenta corriente de la balanza de pagos, mediante las siguientes ecuaciones que resultan de integrar la relación de largo plazo estimada:

Periodo 1950-1983

$$
\underbrace{\ln \left(\frac{Y_{t}}{Y_{t-1}}\right)}_{\text {Tasa teórica }}=\underbrace{0.033+0.297 \ln \left(\frac{X_{t}}{X_{t-1}}\right)}_{\text {Tasa estimada }}
$$

Periodo 1984-1990

$$
\underbrace{\ln \left(\frac{Y_{t}}{Y_{t-1}}\right)}_{\text {Tasa teórica }}=\underbrace{0.033+0.266 \ln \left(\frac{X_{t}}{X_{t-1}}\right)}_{\text {Tasa estimada }}
$$

Periodo 1991-2016

$$
\underbrace{\ln \left(\frac{Y_{t}}{Y_{t-1}}\right)}_{\text {Tasa teórica }}=\underbrace{0.029+0.266 \ln \left(\frac{X_{t}}{X_{t-1}}\right)}_{\text {Tasa estimada }}
$$

Con las tasas de crecimiento teóricas se puede obtener la serie del producto que "equilibra" la cuenta corriente de la balanza de pagos (Jiménez, 1988 y 
2009). Para ello se identificó un año base en cada uno de los periodos. Los años elegidos fueron 1959 y 1990 , ya que en esos años la balanza comercial y de servicios alcanzó un valor cercano a 0 como porcentaje del PIB. También en el año 2003 se registra una balanza aproximadamente igual a 0, pero el empalme de la serie dio un mejor resultado en el sentido económico con los años anteriores.

Con los años base y las tasas teóricas de crecimiento se obtiene el producto de equilibrio de la balanza de pagos. El cuadro 15 y la gráfica 11 muestran que el producto de equilibrio de la balanza de pagos sigue el comportamiento de las exportaciones. En 1950-1982, el PIB y las exportaciones crecen a tasas similares: 4.6 y 4.9\%, respectivamente. En 1983-1990, ambas decrecen. Después, en 1991-2016, crecen a las tasas de 4.7 y $6.6 \%$, respectivamente.

Cuadro 15. Tasas de crecimiento. Promedio anual

\begin{tabular}{lcccccccc}
\hline Periodo & \multicolumn{2}{c}{$P I B^{*}$} & \multicolumn{2}{c}{$P I B$} & \multicolumn{2}{c}{ Exportaciones } & \multicolumn{2}{c}{ Importaciones } \\
\cline { 2 - 9 } & $g_{Y^{*}}$ & $\sigma_{\gamma^{*}}$ & $g_{\gamma^{*}}$ & $\sigma_{Y}$ & $g_{x}$ & $\sigma_{x}$ & $g_{M}$ & $\sigma_{M}$ \\
\hline $1950-1982$ & 0.048 & 0.025 & 0.046 & 0.03 & 0.049 & 0.097 & 0.064 & 0.143 \\
$1983-1990$ & -0.023 & 0.049 & -0.006 & 0.09 & -0.011 & 0.139 & -0.027 & 0.175 \\
$1991-2016$ & 0.045 & 0.012 & 0.047 & 0.03 & 0.066 & 0.058 & 0.069 & 0.107 \\
\hline
\end{tabular}

Notas: * se denomina PIB al producto que equilibra la balanza de pagos; ${ }^{\star \star} g_{n}$ denota la tasa de crecimiento para la variable $\mathrm{n}$ en el periodo correspondiente; ${ }^{\star \star \star} \sigma_{\mathrm{n}}$ denota la desviación estándar de las tasas de crecimiento para la variable $\mathrm{n}$ en el periodo correspondiente.

Fuente: estimaciones con base en datos del BCRP. Elaboración propia.

Grática 11. Perú: producto de equilibrio de la balanza de pagos determinado por las exportaciones (Millones de soles de 2007, en logaritmos)

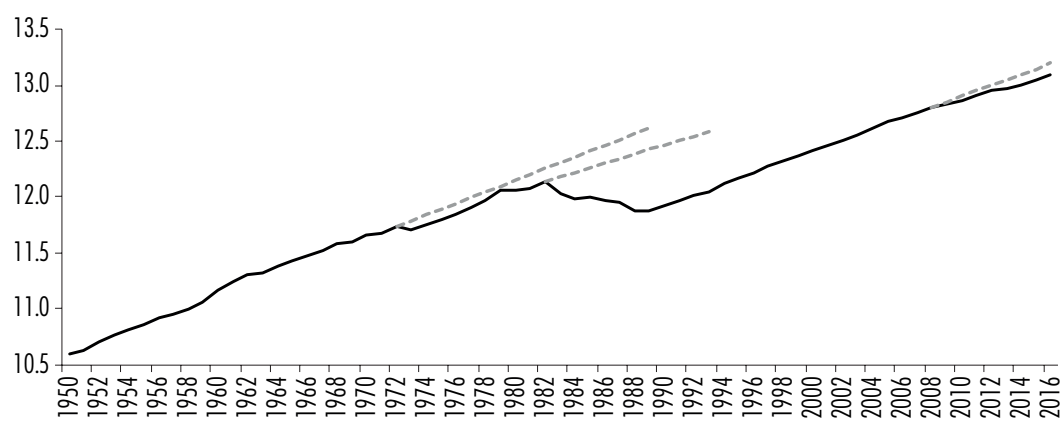

Fuente: estimaciones con base en datos del BCRP. Elaboración propia. 
Finalmente, con la serie de producto observado y el producto que equilibra la balanza de pagos, se obtiene la brecha del producto (véase gráfica 12 ).

Gráfica 12. Perú: ciclo económico (1950-2016) (porcentajes)

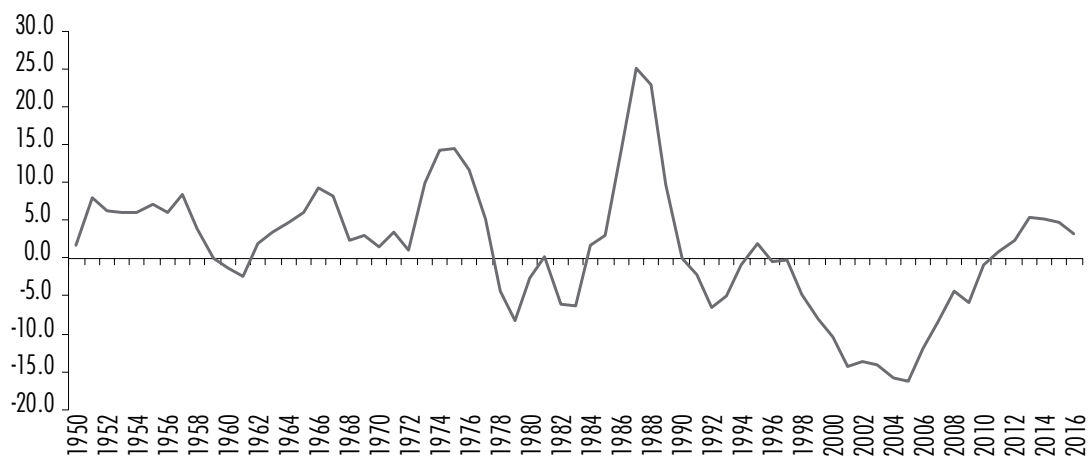

Fuente: estimaciones con base en datos del BCRP. Elaboración propia.

Esta gráfica replica aproximadamente los ciclos obtenidos con el modelo de Shaikh y Moudud (2004) en el periodo 1950-1990. El producto de equilibrio de la balanza de pagos está por encima del producto observado durante los años de 1996-2010, y en el periodo de 2011-2016 ocurre lo contrario. Cuando el producto que equilibra la balanza de pagos se encuentra por encima del nivel del рів observado, se requieren niveles de producción más altos para equilibrar la cuenta corriente y compensar la fuerte penetración de importaciones. En gran parte de estos años la cuenta corriente es deficitaria (véase Apéndice).

Por otra parte, el producto per cápita de equilibrio de la balanza de pagos también muestra dos tipos de comportamiento (véase gráfica 13). En el periodo 1950-1982 crece sostenidamente, aunque presenta un ligero bache en los años de 1970-1976. De 1983 en adelante decrece hasta alcanzar un mínimo en 1989 después del cual crece hasta alcanzar en 2016 un nivel similar al registrado en 1978. 
Gráfica 13. Perú: producto de equilibrio de la balanza de pagos entre la PEA Ocupada

(Soles de 2007 por trabajador)

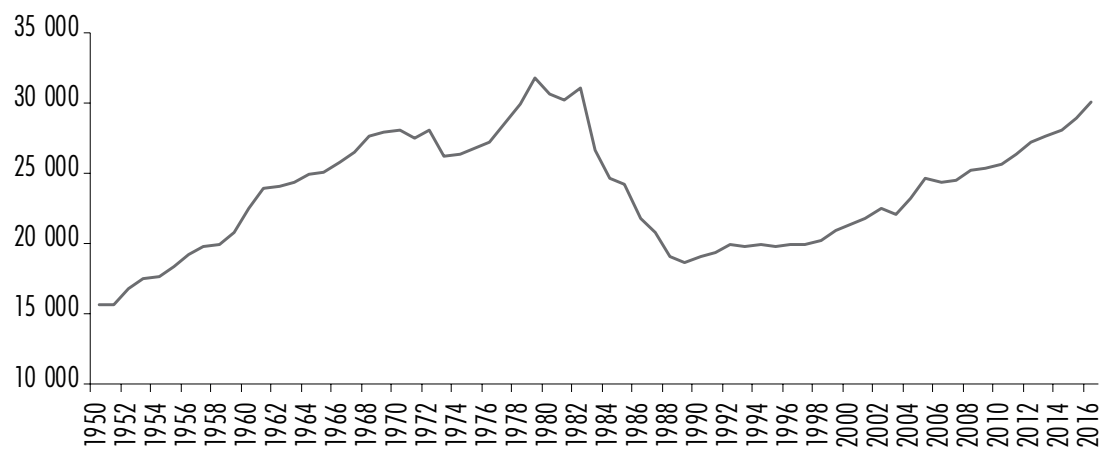

Fuente: estimaciones con base en datos del BCRP y del INEl. Elaboración propia.

\section{PRODUCTO POTENCIAL DETERMINADO POR FACTORES DE OFERTA: EL MODELO DE SOLOW-SWAN}

Para el modelo neoclásico la máxima producción de largo plazo (producto potencial) se alcanza con el pleno uso de los factores de producción, capital y trabajo; es entonces, un producto de pleno empleo de los factores. Se estima este producto potencial a partir de la siguiente ecuación:

$$
\operatorname{Ln} Y_{t}=\alpha \operatorname{Ln} K_{t}+(1-\alpha) \operatorname{Ln} N_{t}+a_{t}
$$

donde $\operatorname{Ln} Y_{t}$ es el logaritmo del producto (РIB); $\operatorname{Ln} K_{t}$ y $\operatorname{Ln} N_{t}$ son los logaritmos de los factores de producción stock de capital (SKA) y mano de obra (PEA); y $(1-\alpha)$ es la elasticidad del producto con respecto al factor trabajo que usualmente se identifica con la participación del ingreso de los trabajadores en la producción. La elasticidad del stock de capital con respecto al producto es $\alpha$. La productividad total de los factores (PTF), que presenta valores con la misma periodicidad que los factores de producción respectivos, es $a_{t}$. La ecuación descrita representa una función de producción con rendimientos constantes a escala.

La estimación de la ecuación (14) como expresión de una relación de largo plazo, se hizo de acuerdo con la metodología de Jiménez (2011), resumida en las siguientes etapas: 
a) Se calcula la participación de los factores de producción capital y trabajo mediante el método de cointegración de Johansen y Juselius (1990), bajo el supuesto que la relación entre la producción y los factores de producción es de largo plazo y exhibe rendimientos constantes a escala. El factor trabajo, en este caso, es el número de trabajadores.

b) Con las participaciones estimadas y los factores de producción, se calcula el PIB explicado exclusivamente por el crecimiento de estos factores $P I B_{f a c}$. Para este cálculo se estima la serie de empleo tendencial $\left(\operatorname{Ln} N_{t}^{*}\right)$ mediante el filtro Hodrick-Prescott. ${ }^{5}$ No se filtra el stock de capital (Antón, 2008). La plena utilización del stock de capital, corresponde a la máxima producción. Se supone que no hay restricción por el lado de la oferta de trabajo disponible.

c) La PTF se calcula como el residuo o diferencia del piB observado y explicado por los factores de producción. La PTF es la que se denomina $a_{t}$.

d) La tendencia de la PTF, $a_{t}$, se estima con el filtro Hodrick-Prescott.

e) Finalmente, el producto potencial $Y_{t}^{*}$ se obtiene como una función de la tendencia de la productividad conjunta de factores $a_{t}^{*}$, del stock de capital $\left(\ln K_{t}\right)$, y del empleo potencial $\left(\operatorname{Ln} N_{t}^{*}\right)$.

La ecuación del producto potencial será entonces:

$$
\operatorname{Ln} Y_{t}^{*}=\alpha \operatorname{Ln} K_{t}+(1-\alpha) \operatorname{Ln} N_{t}^{*}+a_{t}^{*}
$$

Para estimar la ecuación de cointegración hay que comprobar que la serie del factor trabajo (PEA) en logaritmos es integrada de orden 1, como lo son los logaritmos de las series de PIB y stock de capital. En el cuadro 16 se muestra los resultados de aplicar el respectivo test de ADF. La serie es integrada de orden 1 con intercepto y, con intercepto y tendencia. Además, la serie en primeras diferencias, con intercepto, es estacionaria. En consecuencia, ya se puede estimar la ecuación de cointegración entre los logaritmos del PIB y de las variables stock de capital y trabajo.

5 Se utiliza el filtro Hodrick-Prescott con un $1=39$, debido a que los ciclos más importantes tienen una longitud mínima de 9 años y longitudes mayores que se acercan a 12 ańos (Jiménez, 2011). 
Cambio técnico, productividad y estimaciones alternativas del producto de largo plazo

Cuadro 16. Prueba de ráiz unitaria para la PEA de Perú 1950 - 2016

\begin{tabular}{|c|c|c|c|}
\hline Hipótesis nula: & \multicolumn{3}{|c|}{ Log de PEA total tiene raíz unitaria } \\
\hline Exógenas: & \multicolumn{3}{|l|}{ Intercepto } \\
\hline Rezagos: & \multicolumn{3}{|c|}{0 con 5 como máximo, AlC } \\
\hline & & Estadístico $†$ & Probabilidad \\
\hline \multicolumn{2}{|c|}{ Test estadístico Dickey-Fuller Aumentado } & -0.117 & 0.943 \\
\hline \multirow[t]{3}{*}{ Valores críticos } & Nivel 1\% & -3.533 & \\
\hline & Nivel 5\% & -2.906 & \\
\hline & Nivel 10\% & -2.591 & \\
\hline Hipótesis nula: & \multicolumn{3}{|c|}{ Log del PEA total tiene raíz unitaria } \\
\hline Exógenas: & \multicolumn{3}{|c|}{ Intercepto y tendencia } \\
\hline \multirow[t]{2}{*}{ Rezagos: } & \multicolumn{3}{|c|}{2 con 5 como máximo, AlC } \\
\hline & & Estadistico $t$ & Probabilidad \\
\hline \multicolumn{2}{|c|}{ Test estadístico Dickey-Fuller Aumentado } & -2.419 & 0.366 \\
\hline \multirow[t]{3}{*}{ Valores críticos } & Nivel 1\% & -4.108 & \\
\hline & Nivel 5\% & -3.482 & \\
\hline & Nivel 10\% & -3.169 & \\
\hline Hipótesis nula: & \multicolumn{3}{|c|}{ d(Log PEA total) tiene raíz unitaria } \\
\hline Exógenas: & \multicolumn{3}{|l|}{ Intercepto } \\
\hline \multirow[t]{2}{*}{ Rezagos: } & \multicolumn{3}{|c|}{0 con 5 como máximo, AIC } \\
\hline & & Estadístico $†$ & Probabilidad \\
\hline \multicolumn{2}{|c|}{ Test estadístico Dickey-Fuller Aumentado } & -6.987 & 0.000 \\
\hline \multirow[t]{3}{*}{ Valores críticos } & Nivel 1\% & -3.535 & \\
\hline & Nivel 5\% & -2.907 & \\
\hline & Nivel 10\% & -2.591 & \\
\hline
\end{tabular}

Fuente: estimaciones con base en datos del INEl. Elaboración propia. 
En el cuadro 17 contiene los test de traza y de máximo valor propio, que permiten estimar un vector de cointegración. Se rechaza la hipótesis de inexistencia de una ecuación de cointegración. Tanto el test de la traza como el de valor propio indican el mismo resultado. La forma de la ecuación de cointegración que se evaluó cuenta con las variables descritas, una constante y tendencia. Por otro lado, debido al quiebre estructural encontrado en la serie de stock de capital, se incorporó una dummy que toma los valores de 1 desde 1983 hasta 2016, y valores de 0 para el resto de años.

Cuadro 17. Prueba de cointegración de Johansen

\begin{tabular}{|c|c|c|c|c|c|c|}
\hline \multirow[t]{2}{*}{ Rezagos } & \multicolumn{3}{|c|}{ Test de traza } & \multicolumn{3}{|c|}{ Test de máximo valor propio } \\
\hline & Hipótesis nula & $\lambda$ traza & $V C(5 \%)$ & Hipótesis nula & $\lambda$ traza & $V C(5 \%)$ \\
\hline \multirow[t]{3}{*}{4} & $r=0$ & 129.689 & 63.876 & $r=0$ & 74.696 & 32.118 \\
\hline & $r \leq 1$ & 54.993 & 42.915 & $r \leq 1$ & 34.348 & 25.823 \\
\hline & $r \leq 2$ & 20.645 & 25.872 & $r \leq 2$ & 14.009 & 19.387 \\
\hline
\end{tabular}

Fuente: estimaciones con base en datos del INEl. Elaboración propia.

Para la estimación de la ecuación se restringe a 1 la suma de los valores de los coeficientes de los factores capital y trabajo, por el supuesto de rendimientos a escala constantes en todo el periodo. La ecuación de cointegración estimada (véase cuadro 18) reporta un intercepto de 0.755 para el primer periodo 1950-1982, y -0.062 para el segundo. El coeficiente de la tendencia toma el valor de 0.019 , mientras que los coeficientes del stock de capital y la PEA son 0.545 y 0.455 , respectivamente.

Cuadro 18. Ecuación de cointegración de Johansen

\begin{tabular}{lcccccc}
\hline Muestra & $\log ($ PIB) & Constante & Dummy & Tendencia & $\log$ (SKA) & $\log$ (PEA) \\
\hline $1950-2016$ & 1 & 0.755 & -0.816775 & 0.019 & 0.545 & 0.455 \\
& & $(-0.074)$ & $(0.003)$ & $(0.279)$ & $(0.274)$ \\
& & {$[11.0257]$} & {$[5.799]$} & {$[1.984]$} & {$[1.659]$} \\
\hline
\end{tabular}

Fuente: estimaciones con base en datos del INEl. Elaboración propia. 
A los residuos obtenidos de la ecuación se les aplica el test de raíz unitaria de ADF, en este caso considerando como exógeno el intercepto y con un rezago. Los resultados obtenidos muestran que se rechaza la existencia de raíz unitaria en los residuos al 5\% de significancia (véase cuadro 19). Con ello y los resultados anteriores se concluye que existe una relación de cointegración entre las variables consideradas.

Cuadro 19. Test de raíz unitaria en residuos

\begin{tabular}{|c|c|c|c|}
\hline Hipótesis nula: & \multicolumn{3}{|c|}{ Residuos con raíz unitaria } \\
\hline Exógenas: & \multicolumn{3}{|l|}{ Intercepto } \\
\hline Rezagos: & \multicolumn{3}{|c|}{1 con 5 como máximo, AlC } \\
\hline & & Estadístico $t$ & Probabilidad \\
\hline \multicolumn{2}{|c|}{ Test estadístico Dickey-Fuller Aumentado } & -3.959 & 0.003 \\
\hline \multirow[t]{3}{*}{ Valores críticos } & Nivel 1\% & -3.550 & \\
\hline & Nivel 5\% & -2.914 & \\
\hline & Nivel 10\% & -2.595 & \\
\hline
\end{tabular}

Fuente: estimaciones con base en datos del INEl. Elaboración propia.

Por último, se tiene que comprobar que la relación de cointegración es estable (véase cuadro 20). En el modelo de corrección de errores estimado con el método de Johansen y Juselius (1990), el término de corrección del error, que es el coeficiente que multiplica a los residuos de la ecuación de cointegración, tiene el signo negativo esperado y es estadísticamente significativo. La relación de largo plazo entre el producto y los factores de producción es, entonces, estable. La tasa de ajuste de las desviaciones de cada periodo es de 0.206. 
Félix Jiménez

Cuadro 20. Modelo de corrección de errores

\begin{tabular}{|c|c|c|c|c|}
\hline \multirow{2}{*}{$\begin{array}{l}\text { Variable dependiente: } \\
\text { Método: }\end{array}$} & \multicolumn{4}{|l|}{$d \log (P \mid B)$} \\
\hline & \multicolumn{4}{|c|}{ Mínimos Cuadrados Ordinarios } \\
\hline Muestra: & \multicolumn{4}{|l|}{$1955-2015$} \\
\hline Observaciones: & \multicolumn{4}{|l|}{62} \\
\hline Variable & Coeficiente & Error estándar & Estadistico ${ }^{\dagger}$ & Probabilidac \\
\hline Constante & 0.107 & 0.028 & 3.874 & 0.000 \\
\hline $\mathrm{ECT}_{\mathrm{t}-\mathrm{l}}$ & -0.206 & 0.056 & -3.694 & 0.001 \\
\hline$d\left(\log P B_{+-1}\right)$ & 0.527 & 0.144 & 3.660 & 0.000 \\
\hline$d\left(\log \mathrm{PlB}_{\mathrm{F}-2}\right)$ & -0.031 & 0.196 & -0.158 & 0.875 \\
\hline$d\left(\log \mathrm{PIB}_{\mathrm{F}-3}\right)$ & 0.446 & 0.191 & 2.333 & 0.024 \\
\hline$d\left(\log _{P \mid B} B_{+-4}\right)$ & -0.112 & 0.184 & -0.610 & 0.545 \\
\hline$d\left(\log S K A_{t-1}\right)$ & -0.942 & 0.855 & -1.101 & 0.276 \\
\hline$d\left(\log S A_{t-2}\right)$ & -2.013 & 1.307 & -1.540 & 0.131 \\
\hline$d\left(\log S K A_{+3}\right)$ & 2.804 & 1.301 & 2.155 & 0.036 \\
\hline$d\left(\log S K A_{+4}\right)$ & -1.870 & 0.810 & -2.307 & 0.025 \\
\hline$d\left(\log P E A 0_{+-1}\right)$ & -0.385 & 0.371 & -1.038 & 0.304 \\
\hline$d\left(\log P E A 0_{+2}\right)$ & -0.785 & 0.353 & -2.226 & 0.031 \\
\hline$d\left(\log P E A 0_{+-3}\right)$ & -0.175 & 0.367 & -0.478 & 0.634 \\
\hline$d\left(\log P A 0_{t-4}\right)$ & -0.108 & 0.378 & -0.285 & 0.777 \\
\hline $\mathrm{d}\left(\log _{\mathrm{DUM} 83_{+-1}}\right)$ & 0.178 & 0.048 & 3.716 & 0.001 \\
\hline $\mathrm{d}\left(\log _{\mathrm{DUM}} 83_{+2}\right)$ & 0.053 & 0.053 & 0.999 & 0.323 \\
\hline $\mathrm{d}\left(\log \mathrm{DUM} 83_{+-3}\right)$ & 0.158 & 0.050 & 3.186 & 0.003 \\
\hline 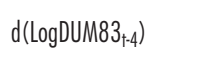 & 0.120 & 0.054 & 2.228 & 0.031 \\
\hline$R^{2}$ ajustado & 0.406 & & & \\
\hline
\end{tabular}

Fuente: estimaciones con base en datos del INEl. Elaboración propia. 


\section{Producto potencial y productividad total de factores}

Para calcular la productividad total de factores de largo plazo $\left(a_{t}^{*}\right)$, en primer lugar, se obtiene por diferencia la parte del pIB que no es explicado por los factores capital y trabajo; en segundo lugar, se filtra la serie de productividad total de factores $\left(a_{t}\right)$ utilizando el filtro Hodrick-Prescott sin restricción de colas y con restricción de colas.

El producto potencial será la suma del producto explicado por los factores de producción y la productividad total de factores de largo plazo. En consecuencia, la ecuación del producto potencial (Ріврот) con sus respectivos coeficientes tiene la forma siguiente:

$$
\begin{aligned}
& \operatorname{Ln} Y_{t}^{*}=\alpha \operatorname{Ln} K_{t}+(1-\alpha) \operatorname{Ln} N_{t}^{*}+a_{t}^{*} \\
& \operatorname{LN}(P I B T O T)=0.545 \operatorname{Ln}\left(S K A_{t}\right)+0.455 \operatorname{Ln}\left(P E A_{t}^{*}\right)+a_{t}^{*}
\end{aligned}
$$

Como puede verse en la gráfica 14 , el producto potencial crece sostenidamente hasta fines de la década de 1960, luego se desacelera hasta estancarse en la década de 1980. Claramente hay una reducción del nivel del producto potencial que recién a principios de la década de 1990 empieza a recuperarse.

Por su parte, la PTF aumenta de forma sostenida desde 1950 hasta los años de 1974-1975 en los que alcanza un máximo (véase gráfica 15). De 1975 a 1992 registra una tendencia decreciente. En 1992 alcanza un mínimo, con un valor similar al de 1951. Este enorme retroceso ocurre precisamente en los años de ajustes fiscales y monetarios, y cubre la denominada década perdida de América Latina que también lo fue para la economía peruana. De 1992-2002, la PTF registra una tendencia ligeramente creciente. En realidad, esta tendencia es insignificante; lo que indica que el crecimiento del PIB de los años de 1993 a 1997 fue básicamente por acumulación de factores. Luego crece sostenidamente desde 2003 hasta 2016. Este fue un periodo de alto crecimiento económico asociado a los altos precios de los minerales que exporta el país.

La gráfica 16 muestra el ciclo económico. Cuando se aplica el filtro Hodrick-Prescott a la productividad total de factores sin corrección de colas, los dos últimos años son de recesión económica (véase gráfica 16). Si se aplica el filtro con corrección de colas, la economía estaría atravesando un periodo de auge desde 2013 (véase gráfica 17). 
Félix Jiménez

Gráfica 14. Perú: producto potencial (millones de soles de 2007, en logaritmos)

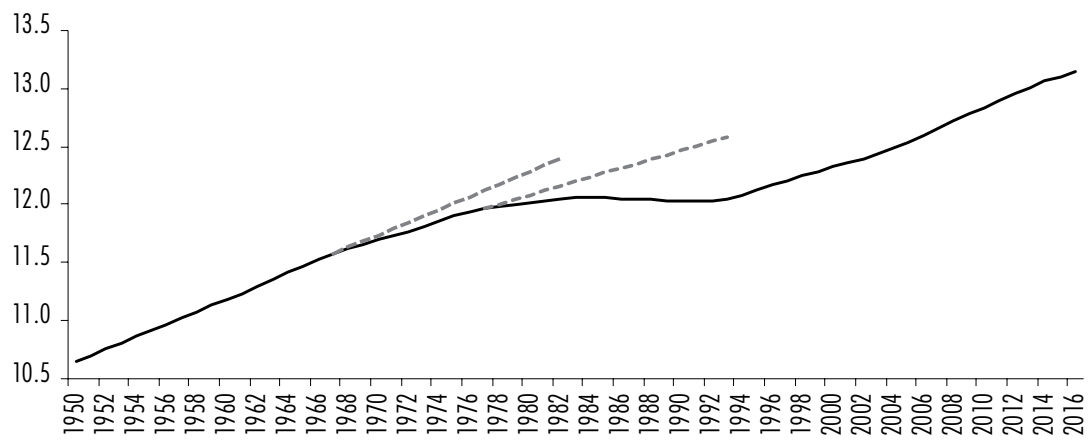

Fuente: estimaciones con base en datos del INEl. Elaboración propia.

Gráfica 15. Perú: productividad total de factores (1950-2016) (en logaritmos)

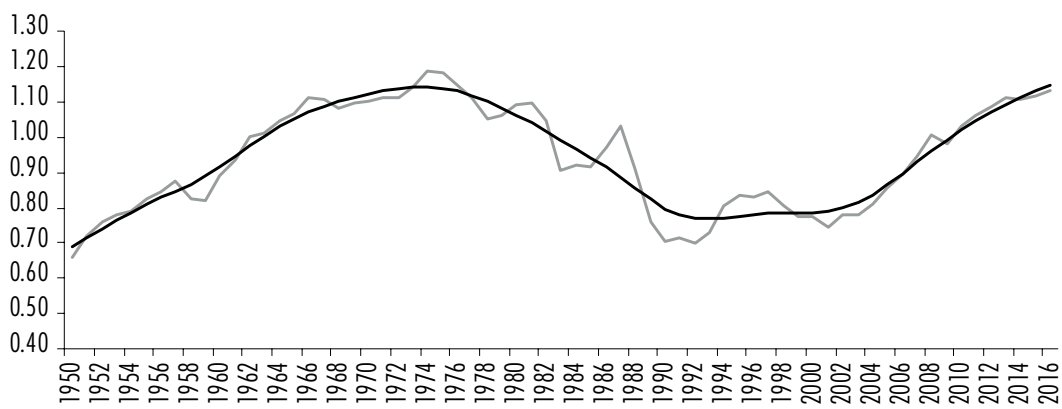

— PTF - PTF tendencial

Fuente: estimaciones con base en datos del INEl. Elaboración propia.

Gráfica 16. Perú: ciclo económico (1950-2016) (porcenties)

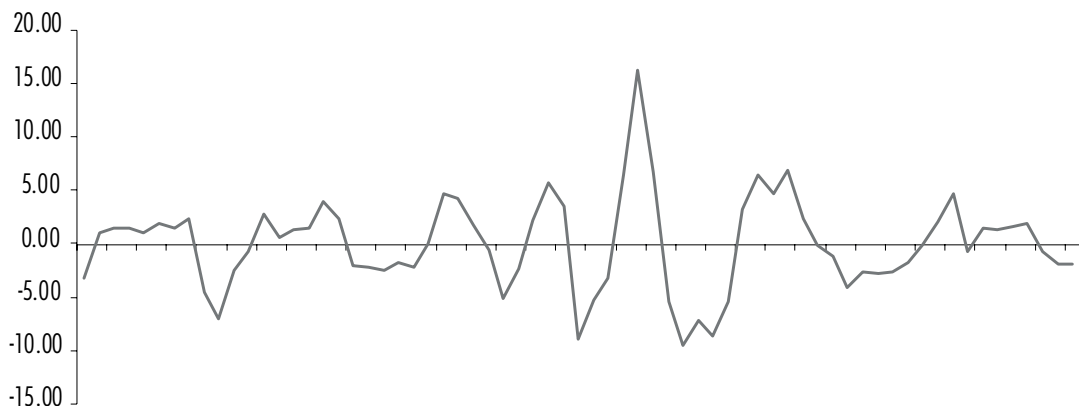

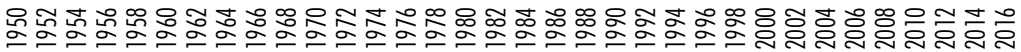
Fuente: estimaciones con base en datos del INEl. Elaboración propia. 
Gráfica 17. Perú: producción de largo plazo determinado por demanda y por oferta

(Millones de soles de 2007, en logaritmos)

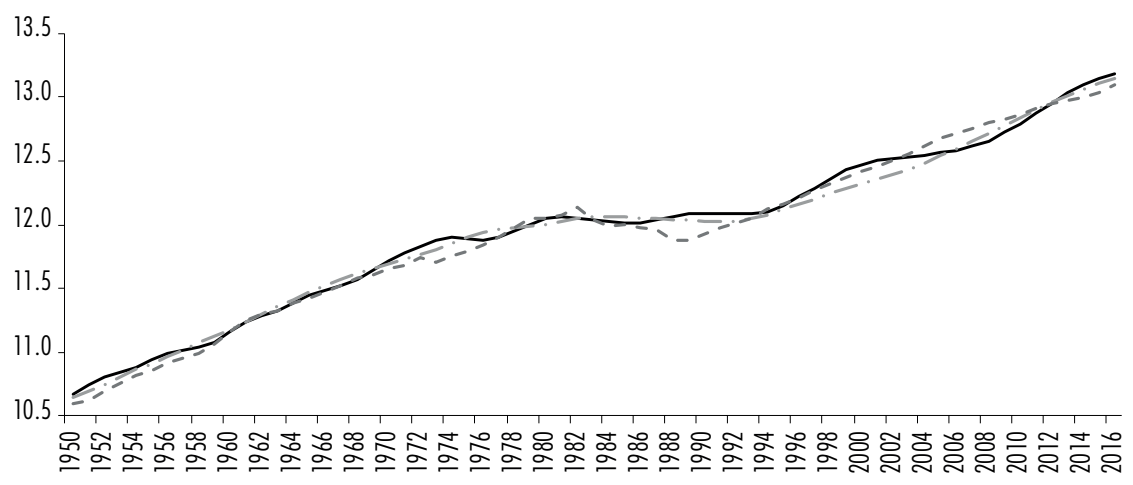

_ Capacidad económica productiva _- Producto potencial _ _ Producto de equilibrio de la balanza de pagos

Fuente: estimaciones con base en datos del INEl. Elaboración propia.

\section{CONCLUSIONES}

La "capacidad económica productiva" estimada, siguiendo a Shaikh y Moudud (2004), representa la máxima producción deseada por los inversionistas y está determinada por la demanda. El propio proceso de acumulación de capital y el cambio técnico responden al comportamiento de la demanda agregada. En tanto que, el "producto potencial" -estimado siguiendo a Solow y Swan-, también representa la máxima producción de largo plazo, sólo que en este caso se determina por el pleno uso de los factores de producción disponibles y la tecnología existente. Se supone que la demanda sólo influye en las fluctuaciones de la producción. Finalmente, también se estimó el "producto de equilibrio de la balanza de pagos", siguiendo a Thirlwall (1979).

Los tres tipos de producción de largo plazo pueden verse en la gráfica 17. Se observa que siguen una tendencia similar, pero no coinciden en varios tramos del periodo de análisis 1950-2016.

Tampoco coinciden los respectivos ciclos económicos. Los cuadros 21 y 22 muestran las diferencias estadísticas de estos ciclos. Todos los ciclos económicos son estacionarios, de acuerdo con el estadístico del test ADF (véase cuadro 21). El que tiene la mayor volatilidad es el estimado a partir de Thirlwall (1979). De otro lado, los ciclos estimados con los métodos de Shaikh y Moudud y el neoclásico son los únicos que están altamente correlacionados. 
Félix Jiménez

Cuadro 21. Estadísticas descriptivas de los ciclos económicos

\begin{tabular}{lcccc}
\hline \multicolumn{1}{c}{ Método } & Media & Desviación estándar & Coeficiente de variación & ADF \\
\hline Shaikh-Moudud & 0.984 & 0.071 & 0.072 & $-4.667^{0}$ \\
Thirlwall & 1.015 & 0.085 & 0.083 & $-3.871^{\mathrm{b}}$ \\
Neoclásico & 1.001 & 0.041 & 0.041 & $-5.265^{\mathrm{c}}$ \\
\hline
\end{tabular}

Notas: ${ }^{a} 1 \%$ de significancia con 1 rezago; ${ }^{b} 1 \%$ de significancia con 1 rezago; ${ }^{c} 1 \%$ de significancia con 1 rezago.

Fuente: estimaciones con base en datos del INEl y BCRP. Elaboración propia.

Cuadro 22. Correlación entre los ciclos económicos $\left(^{\star}\right)$

\begin{tabular}{lcccc}
\hline & Shaikh y Moudud & Thirlwall & Thirlwall Con T.I. & Neoclásico \\
\hline Shaikh y Moudud & 1.000 & 0.542 & 0.226 & 0.699 \\
& & $(5.205)$ & $(1.870)$ & $(7.880)$ \\
\multirow{2}{*}{ Thirlwall } & 0.542 & 1.000 & 0.644 & 0.515 \\
& $(5.205)$ & & $(6.779)$ & $(4.848)$ \\
Neoclásico & 0.699 & 0.515 & 0.485 & 1.000 \\
& $(7.880)$ & $(4.848)$ & $(4.468)$ & \\
\hline
\end{tabular}

Nota: $\left.{ }^{\star}{ }^{\star}\right)$ Los estadísticos-t se encuentran entre paréntesis: $t=r \sqrt{\frac{n-2}{1-r^{2}}}$

Fuente: estimaciones con base en datos del INEl y BCRP. Elaboración propia.

\section{APÉNDICE}

\begin{tabular}{lcccccccc}
\hline Año & PIB & SKA $^{b}$ & XbSs $^{c}$ & PEAO $^{d}$ & BCC $^{e}$ & PIB $_{\text {CEP }}$ & PIB $_{B P}$ & PIB $_{\text {POT }}$ \\
\hline 1950 & 40920 & 101953 & 5943 & 2584 & 11.6 & 42922 & 40201 & 42267 \\
1951 & 44711 & 104009 & 5824 & 2642 & 0.1 & 46688 & 41281 & 44261 \\
1952 & 47347 & 107680 & 6692 & 2646 & -49.3 & 49376 & 44444 & 46683 \\
1953 & 50085 & 112368 & 7328 & 2700 & -63.5 & 51433 & 47170 & 49381 \\
1954 & 52762 & 117618 & 7826 & 2816 & -18.2 & 53265 & 49691 & 52238 \\
1955 & 55858 & 121428 & 8213 & 2835 & -95.1 & 56634 & 52076 & 54768 \\
1956 & 58484 & 126325 & 8889 & 2881 & -107.7 & 59342 & 55077 & 57599 \\
1957 & 62371 & 132759 & 9147 & 2912 & -155.4 & 60985 & 57384 & 60905
\end{tabular}




\begin{tabular}{|c|c|c|c|c|c|c|c|c|}
\hline Año & $P \mid B^{a}$ & $S K A^{b}$ & $X_{b s s}{ }^{c}$ & PEAO ${ }^{d}$ & $B C C^{e}$ & $P I B^{f}{ }_{C E P}$ & $P I B_{B P}$ & PIB ${ }_{\text {POT }}^{h}$ \\
\hline 1958 & 61706 & 139920 & 9210 & 2996 & -122.3 & 62333 & 59402 & 64576 \\
\hline 1959 & 63653 & 145822 & 10416 & 3061 & -40.0 & 65092 & 63653 & 68263 \\
\hline 1960 & 69946 & 149122 & 13388 & 3146 & 7.9 & 70477 & 70853 & 71694 \\
\hline 1961 & 75085 & 152912 & 15797 & 3227 & -12.4 & 75913 & 76886 & 75621 \\
\hline 1962 & 82620 & 158771 & 16862 & 3369 & -37.2 & 79837 & 80984 & 80399 \\
\hline 1963 & 86196 & 165760 & 16559 & 3417 & -81.8 & 83090 & 83211 & 85671 \\
\hline 1964 & 91840 & 171596 & 17701 & 3512 & 14.1 & 87887 & 87683 & 90697 \\
\hline 1965 & 97003 & 177222 & 18215 & 3641 & -142.5 & 93378 & 91356 & 95618 \\
\hline 1966 & 104995 & 184773 & 19067 & 3720 & -228.4 & 97434 & 95668 & 100965 \\
\hline 1967 & 109040 & 193737 & 20189 & 3801 & -281.9 & 100575 & 100525 & 106525 \\
\hline 1968 & 109206 & 201154 & 22181 & 3867 & -40.6 & 105781 & 106795 & 111437 \\
\hline 1969 & 113044 & 206184 & 21790 & 3933 & 1.3 & 114026 & 109744 & 115540 \\
\hline 1970 & 116849 & 211568 & 23035 & 4108 & 165.3 & 122660 & 115260 & 119729 \\
\hline 1971 & 122213 & 218333 & 22360 & 4288 & -64.5 & 130520 & 118022 & 124340 \\
\hline 1972 & 126463 & 226546 & 24439 & 4466 & -63.8 & 137444 & 125190 & 129340 \\
\hline 1973 & 134401 & 235336 & 19914 & 4641 & -269.9 & 144420 & 121690 & 134330 \\
\hline 1974 & 147017 & 248555 & 20950 & 4839 & -906.7 & 147017 & 127623 & 140269 \\
\hline 1975 & 153340 & 266526 & 21432 & 4956 & -1566.0 & 145388 & 132736 & 146942 \\
\hline 1976 & 155559 & 285025 & 22167 & 5095 & 1107.0 & 144521 & 138506 & 152901 \\
\hline 1977 & 156102 & 299894 & 25038 & 5198 & -816.4 & 148191 & 148361 & 157010 \\
\hline 1978 & 151977 & 312704 & 28277 & 5303 & - 164.0 & 154598 & 158911 & 159950 \\
\hline 1979 & 158194 & 323553 & 32989 & 5421 & 953.6 & 163679 & 171863 & 161956 \\
\hline 1980 & 167596 & 336258 & 29747 & 5623 & -394.2 & 171839 & 172167 & 164049 \\
\hline 1981 & 176901 & 354480 & 28973 & 5847 & -2197.1 & 173200 & 176469 & 167002 \\
\hline 1982 & 176507 & 377327 & 31856 & 6033 & -2055.3 & 172078 & 187518 & 170403 \\
\hline 1983 & 158136 & 398468 & 28197 & 6335 & -1075.7 & 170075 & 168401 & 172834 \\
\hline 1984 & 163842 & 409665 & 30288 & 6558 & -276.3 & 167422 & 161272 & 172847 \\
\hline 1985 & 167219 & 419970 & 31534 & 6712 & -70.2 & 165536 & 162174 & 172664 \\
\hline 1986 & 182981 & 426867 & 27328 & 7284 & -1473.1 & 164565 & 158513 & 171499 \\
\hline 1987 & 200778 & 438002 & 26351 & 7559 & -2066.8 & 168473 & 156273 & 170654 \\
\hline 1988 & 181822 & 453161 & 24601 & 7598 & -1830.2 & 174086 & 144503 & 169906 \\
\hline 1989 & 159436 & 464678 & 29218 & 7776 & -600.1 & 178174 & 144593 & 168271 \\
\hline
\end{tabular}


Apéndice (continuación)

\begin{tabular}{|c|c|c|c|c|c|c|c|c|}
\hline Año & $P I B^{a}$ & $S K A^{b}$ & $X_{b s s}{ }^{c}$ & PEAO ${ }^{d}$ & $B C C^{e}$ & $P I B_{\text {CEP }}^{f}$ & $P I B_{B P}$ & $P I B^{h_{P O I}}$ \\
\hline 1990 & 151492 & 472171 & 25797 & 7932 & -1459.4 & 178290 & 151492 & 166638 \\
\hline 1991 & 154854 & 479820 & 27297 & 8201 & -1518.7 & 178438 & 158163 & 166481 \\
\hline 1992 & 154017 & 487624 & 28482 & 8296 & -1916.1 & 178615 & 164512 & 168040 \\
\hline 1993 & 162093 & 495396 & 29371 & 8669 & -2464.3 & 178650 & 170581 & 171257 \\
\hline 1994 & 182044 & 505891 & 35057 & 9236 & -2701.3 & 180981 & 183894 & 176289 \\
\hline 1995 & 195536 & 523896 & 37001 & 9744 & -4624.5 & 189822 & 191861 & 183433 \\
\hline 1996 & 201009 & 547864 & 40282 & 10120 & -3643.9 & 203971 & 201831 & 191870 \\
\hline 1997 & 214028 & 569959 & 45559 & 10758 & -3368.0 & 216370 & 214486 & 199794 \\
\hline 1998 & 213190 & 597035 & 48099 & 11082 & -3336.0 & 233468 & 223795 & 208398 \\
\hline 1999 & 216377 & 623391 & 51774 & 11262 & -1379.9 & 249917 & 234716 & 216845 \\
\hline 2000 & 222207 & 644730 & 55911 & 11573 & -1545.8 & 261273 & 246382 & 224752 \\
\hline 2001 & 223580 & 663259 & 59729 & 11862 & -1202.8 & 269563 & 257884 & 233013 \\
\hline 2002 & 235773 & 678374 & 64237 & 12034 & -1093.7 & 274145 & 270406 & 241972 \\
\hline 2003 & 245593 & 693082 & 68194 & 12837 & -929.9 & 278057 & 282558 & 252472 \\
\hline 2004 & 257770 & 707825 & 78580 & 13059 & 59.8 & 281744 & 301769 & 264749 \\
\hline 2005 & 273971 & 723389 & 90527 & 13124 & 1158.9 & 285978 & 322266 & 278955 \\
\hline 2006 & 294598 & 740297 & 91251 & 13683 & 2912.4 & 291251 & 332136 & 294992 \\
\hline 2007 & 319693 & 762298 & 97501 & 14197 & 1520.5 & 301203 & 347660 & 313292 \\
\hline 2008 & 348923 & 786868 & 104429 & 14459 & -5285.5 & 313381 & 364142 & 332809 \\
\hline 2009 & 352584 & 823597 & 103641 & 14758 & -613.9 & 337483 & 373746 & 355314 \\
\hline 2010 & 382380 & 855409 & 105044 & 15090 & -3564.4 & 356546 & 385755 & 376674 \\
\hline 2011 & 407052 & 900215 & 112310 & 15307 & -3373.8 & 389174 & 403853 & 400858 \\
\hline 2012 & 431273 & 945089 & 118819 & 15541 & -6091.0 & 422258 & 421616 & 424583 \\
\hline 2013 & 456366 & 990695 & 117286 & 15684 & -10379.6 & 456435 & 432113 & 447916 \\
\hline 2014 & 467280 & 1034510 & 116282 & 15797 & -9085.9 & 488690 & 443388 & 470345 \\
\hline 2015 & 482797 & 1073007 & 120991 & 15919 & -9525.9 & 514554 & 460844 & 491389 \\
\hline 2016 & 501699 & 1102111 & 132478 & 16197 & -5064.4 & 528816 & 485533 & 510409 \\
\hline
\end{tabular}

Notas: ${ }^{a}$ Producto Interno Bruto, en millones de soles de 2007; ${ }^{b}$ Stock de Capital, en millones de soles de 2007; ' Exportacio-

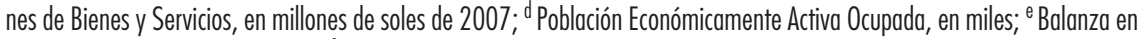
Cuenta Corriente, millones de US\$; ${ }^{\dagger}$ Capacidad Económica Productiva, en millones de soles de 2007; 9 Producto de Equilibrio de la Balanza de Pagos, en millones de soles de 2007; ' Producto Potencial determinado por factores de oferta, en millones de soles de 2007.

Fuente: elaboración propia. 


\section{BIBLIOGRAFÍA}

Antón, A. (2008), El problema al final de la muestra en la estimación del PIB potencial, Documento de Trabajo no. 442, División de Economía, CIDE.

Atesoglu, H. S. (1997), "Economic balance of payments constrained growth model and its implications for the United States", Journal of Post Keynesian Economics, vol. 19, núm. 3.

Ball, L. (2014), Long-term damage from the great recession in OECD countries, Cambridge, National Bureau of Economic Research, Working Paper no. 20185.

Barbosa Filho, N. H. (2005), Estimating potential output: a survey of the alternative methods and their applications to Brazil, Brasilia, Texto para Discussão número 1092, IPEA.

Blanchard, O. (2014), "Where danger lurks", Finance and Development, vol. 51, núm. 3.

Clavijo, P. y Ros, J. (2015), "La Ley de Thirlwall: una lectura crítica”, Investigación Económica, vol. LXXIV, núm. 292, abril-junio.

De Long, J. B. y Summers, L. (1988), "How does macroeconomic policy affect output?", Brookings Pappers on Economic Activity, 2.

Fatás, A. y Summers, L. (2015), The permanent effects of fiscal consolidations, Londres, Centre for Economic Policy Research, Discussion Paper no. 10902.

Gregory, A. y Hansen, B. (1996a), "Residual-based tests for cointegration in models with regime shifts", Journal of Econometrics, vol. 70, núm. 1.

Gregory, A. y Hansen, B. (1996b), "Tests for cointegration in models with regime and trend shifts", Oxford Bulletin of Economics and Statistics, vol. 58 , núm. 3.

Haltmaier, J. (2012), “Do recessions affect potential output?”, International Finance Discussion Papers.

Harrod, R. (1939), "An essay in dynamic theory", The Economic Journal, vol. 49, núm. 193.

Jiménez, F. (1984), "La balanza de pagos como factor limitativo del crecimiento y el desequilibrio estructural externo de la economía peruana", Socialismo y Participación 25.

(1988), Economía peruana: límites internos y externos al crecimiento económico, Lima, Fundación Friedrich Ebert.

(2009), "La economía peruana frente a la crisis y las restricciones al crecimiento económico", en O. Dancourt y F. Jiménez (eds.), Crisis in- 
ternacional. Impactos y respuestas de la política económica en el Perú, Lima, Fondo Editorial Pontificia Univerisidad Católica del Perú.

(2011), "Producto potencial, fuentes del crecimiento y productividad en la economía peruana (1950-2008)", El Trimestre Económico, vol. LXXVIII, núm. 312.

(2017), Crecimiento y desindustrialización prematura en Perú. Un análisis kaldoriano, Pontificia Universidad Católica del Perú, Departamento de Economía, Documento de Trabajo 441, junio.

Johansen, S. y Juselius, K. (1990), "Maximum likelihood estimation and inference on cointegration-with application to the demand for money", Oxford Bulletin of Economics and Statistics, vol. 52, núm. 2.

Kaldor, N. (1957), "A model of economic growth", The Economic Journal, vol. 67, núm. 268.

Martin, R., Munyan, T. y Wilson, B. A. (2015), Potential output and recessions: Are we fooling ourselves?, Washington, D.C., Board of Governors of the Federal Reserve System, International Finance Discussion Papers no. 1145.

McCombie, J. y Thirlwall, A. (1994), Economic growth and the balance-ofpayments constraint, Londres, St. Martin's Press.

Moreno-Brid, J. C. (1998), "On capital flows and the balance of payments constrained growth model”, Journal of Post Keynesian Economics, 21.

Naciones Unidas (1957), Analyses and projections of economic development, VI: The industrial development of Peru, Nueva York.

Reifschneider, D., Wascher, W. y Wilcox, D. (2013), Aggregate supply in the United States: Recent developments and implications for the conduct of monetary policy, Washington DC., International Monetary Fund.

Ros, J. (2004), La teoría del desarrollo y la economía del crecimiento, México, Fondo de Cultura Económica.

(2009), Estancamiento y crisis en la economía mexicana. Trabajo preparado para la XVIII Conferencia Internacional de AfeIEAL, México, Facultad de Economía, UnAM.

(2013), Algunas tesis equivocadas sobre el estancamiento económico de México, México, El Colegio de México-unam.

Shaikh, A. y Moudud, J. (2004), Measuring capacity utilization in OECD countries: A cointegration method, Nueva York, The Levy Economics Institute of Bard College, Working Paper no. 415.

Solow, R. (1956), "A contribution to the theory of economic growth", Quarterly Journal of Economics, vol. 70, núm. 1.

(1988), "Growth theory and after", The American Economic Review, vol. 78 , núm. 3. 
Swan, T. (1956), "Economic growth and capital accumulation", Economic Record, vol. 32, núm. 63.

Thirlwall, A. (1979), "The balance of payments constraint as an explanation of international growth rate differences", Banca Nazionale del Lavoro Quarterly Review, vol. 32, núm. 128. (2003), La naturaleza del crecimiento económico: un marco alternativo para comprender el desempeño de las naciones, México, Fondo de Cultura Económica.

Thirlwall, A. y Hussain, M. (1982), "The balance of payments constraint, capital flows, and growth rate differences between developing countries", Oxford Economic Papers, vol. 34, núm. 3.

Troncoso-Valverde, C. (2004), Structural breaks, cointegration and the domestic demand for chilean wine, Talca, Universidad de Talca, Facultad de Ciencias Empresariales, Working Paper no. 1. 
